\title{
1 Transcription and splicing dynamics during early Drosophila development
}

2

3

5

6

$7 \quad{ }^{1}$ Instituto de Medicina Molecular João Lobo Antunes, Faculdade de Medicina, Universidade de

8 Lisboa, 1649-028 Lisboa, Portugal.

$9{ }^{2}$ Algarve Biomedical Center Research Institute (ABC-RI), Universidade do Algarve, 8005-139 Faro,

10 Portugal.

$11{ }^{3}$ Department of Medical Sciences and Institute for Biomedicine (iBiMED), Universidade de Aveiro, $12 \quad 3810-193$ Aveiro, Portugal.

13

14

RUNNING TITLE: Transcription and splicing dynamics in Drosophila

20 KEYWORDS: splicing kinetics; Pol II pausing; transcription termination; NET-seq; Drosophila melanogaster embryo

22 


\section{ABSTRACT}

25 Widespread co-transcriptional splicing has been demonstrated from yeast to human. However, the 26 precise kinetics of splicing relative to transcription remains unclear, with discrepancies between studies

27 that use different experimental approaches. Here, we adapted native elongating transcript sequencing technology (NET-seq) to measure co-transcriptional splicing dynamics during the early developmental stages of Drosophila melanogaster embryos. Our results reveal the position of RNA polymerase II (Pol II) when both canonical and recursive splicing occur. We found heterogeneity in splicing dynamics, with some RNAs spliced immediately after intron transcription, whereas for other transcripts no splicing was observed over the first 100 nucleotides of the downstream exon. Introns that show splicing completion before Pol II has reached the end of the downstream exon are necessarily intron-defined. We studied the splicing dynamics of both nascent pre-mRNAs transcribed in the early embryo, which have few and short introns, as well as pre-mRNAs transcribed later in embryonic development, which contain multiple long introns. As expected, we found a relationship between the proportion of spliced reads and intron size. However, intron definition was observed at all intron sizes. We further observed that transcription termination was very efficient for isolated genes but that the presence of an overlapping antisense gene was often associated with transcriptional readthrough. Taken together, our data unravels novel dynamic features of Pol II transcription and splicing in the developing Drosophila embryo. 
52

53

54

55

56

57

58

59

60

61

62

63

64

65

66

67

68

69

70

71

72

73

\section{INTRODUCTION}

It is by now largely accepted that splicing can happen, and often does happen, whilst transcription is still in progress (Ameur et al. 2011; Khodor et al. 2011; Beyer and Osheim 1988; Brugiolo et al. 2013; Carrillo Oesterreich et al. 2010; Windhager et al. 2012; Nojima et al. 2015; Alpert et al. 2017). It is also known that the processes of splicing and transcription are tightly interlinked. The RNA Polymerase II (Pol II) elongation rate can affect exon inclusion (Fong et al. 2014; Maslon et al. 2019; Aslanzadeh et al. 2018), and many of the proteins involved in splicing associate with the Pol II large subunit Cterminal domain (CTD) (Hsin and Manley 2012; Emili et al. 2002; David et al. 2011; Görnemann et al. 2011; Morris and Greenleaf 2000; Nojima et al. 2018). Conversely, splicing may also affect transcription, with evidence suggesting that Pol II slows down at exons (Alexander et al. 2010; Carrillo Oesterreich et al. 2010; Jonkers et al. 2014; Mayer et al. 2015; Veloso et al. 2014), potentially to allow for splicing to complete.

The exact timing of intron excision relative to Pol II elongation remains more controversial. A nascent RNA sequencing study (Carrillo Oesterreich et al. 2016) showed that in Saccharomyces cerevisiae, splicing could occur as soon as the 3' splice site had emerged from the transcription machinery, suggesting that splicing may be completed immediately after intron transcription. Previous attempts to determine the duration of splicing in vivo had returned estimates ranging from a few seconds to several minutes (Alpert et al. 2017). The lowest of these estimates, such as the few-second estimate reported in Martin et al. (2013), are consistent with splicing being completed right after the 3' splice site is transcribed.

More recently, two long-read nascent RNA sequencing studies addressed the timing of splicing in metazoa. One reported little immediate splicing in human and Drosophila cultured cell lines, with most splicing occurring when Pol II had elongated thousands of nucleotides past the splice junction (Drexler et al. 2020). Another found that splicing in mouse cells occurred immediately after transcription, when Pol II was within 75-300 nucleotides of 3' splice sites (Reimer et al. 2021). These discrepant results probably reflect biases introduced by the distinct methodological approaches used. Indeed, one study (Drexler et al. 2020) relies on metabolic labelling of newly synthesized RNA with 4sU, while the other extracts RNA from chromatin (Reimer et al. 2021). 
II, the DNA template and the nascent RNA by immunoprecipitation, without crosslinking (Churchman and Weissman 2011; Nojima et al. 2015). Solubilisation of Pol II complexes under native conditions is typically incomplete in metazoan cells (Kimura et al. 1999), but this problem was circumvented by extensive micrococcal nuclease (MNase) digestion of isolated native chromatin (Nojima et al. 2015). Elongation complexes can then be immunoprecipitated using antibodies that specifically recognize the different phosphorylation states of the Pol II CTD. The main advantage of this approach is that it provides global, bidirectional maps of Pol II CTD phosphorylation specific nascent transcripts and coupled RNA processing at single nucleotide resolution. Although initially applied to mammalian cells, and thus termed mNET-seq (Nojima et al. 2015), the technology can be adjusted to any organism, as recently illustrated in plants (Zhu et al. 2018). Here, we have adapted NET-seq to study the dynamic properties of transcription and co-transcriptional splicing during early stages of development in Drosophila melanogaster embryos. An advantage of this model system is that compared to human, the Drosophila genome is more compact (Graveley et al. 2011) and thus the coverage of NET-seq reads on intragenic regions is higher. Another advantage of the Drosophila model is that genes transcribed initially in the embryo have few and short introns (like yeast genes), whereas genes transcribed later contain multiple long introns (more similar to human genes). An additional feature of Drosophila is the presence of many genes with exceptionally long introns that are subdivided by a non-canonical mechanism termed recursive splicing (Duff et al. 2015; Joseph et al. 2018; Pai et al. 2018). For all these reasons, Drosophila is an attractive model to study co-transcriptional splicing dynamics. Drosophila early development is characterized by rapid mitotic cycles that lack cytokinesis, resulting in nuclear proliferation in a syncytial cytoplasm (Campos-Ortega 1985; Laver et al. 2015).

103 During these initial mitotic divisions, which impose significant constraints on transcription and splicing 104 (Shermoen and O'Farrell 1991; Rothe et al. 1992; Guilgur et al. 2014; Martinho et al. 2015; Sandler et al. 2018; Kwasnieski et al. 2019), the embryo largely relies on maternally deposited mRNAs. Subsequently, the duration of the cell cycle is progressively expanded, membranes form between the nuclei and segregate them into cells, and the zygotic transcriptome starts to be fully expressed (Campos- 
bioRxiv preprint doi: https://doi.org/10.1101/2020.11.05.367888; this version posted July 25, 2021. The copyright holder for this preprint

(which was not certified by peer review) is the author/funder, who has granted bioRxiv a license to display the preprint in perpetuity. It is made available under aCC-BY-NC-ND 4.0 International license.

108 Ortega 1985; Yuan et al. 2016; Blythe and Wieschaus 2015). By performing $d$ NET-seq in the

109 developing Drosophila embryo, we have unravelled novel dynamic features of Pol II transcription and

110 splicing.

111 
RESULTS

\section{Native elongating transcript sequencing in Drosophila embryos (dNET-seq)}

115 Our first task was to adapt the NET-seq technology for use in Drosophila embryos (henceforth referred mitotic cycle 14 (stage 5), when membranes form between the nuclei, which are evenly distributed in a monolayer under the egg surface, segregating them into separate cells (Campos-Ortega 1985; Laver et al. 2015). The majority of late embryos were in the late stage of germ-band extension (stage 10; Fig 1C), when the embryo trunk (also known as the germ-band) elongates in the antero-posterior axis and narrows in the dorso-ventral axis (Campos-Ortega 1985; Laver et al. 2015). to purify the chromatin fraction from manually sorted embryos, solubilize the transcription complexes with MNase digestion and immunoprecipitate Pol II with antibodies. We used rabbit polyclonal antibodies raised against synthetic peptides of the YSPTSPS repeat of the CTD of the largest Pol II subunit in Saccharomyces cerevisiae, phosphorylated at either S5 (ab5131 Abcam) or S2 (ab5095 in Drosophila melanogaster (Boija et al. 2017; Vizcaya-Molina et al. 2018; Akhtar et al. 2019; Dahlberg et al. 2015; Lee et al. 2015; Yan et al. 2014; Arzate-Mejía et al. 2020). was first converted to a 5' phosphate by T4 polynucleotide kinase (Fig 1D). RNA was then purified from the immunoprecipitated Pol II complexes and size-selected using an RNA purification kit procedure that combines a unique buffer system with a column technology (see the methods section for more detail). RNAs with a size above 60 nucleotides (nt) were used for subsequent ligation of specific adapters to the 5' $\mathrm{P}$ and 3' $\mathrm{OH}$ ends of each RNA fragment followed by PCR-based preparation of a cDNA library for high-throughput Illumina sequencing (Fig 1D). After sequencing, adapter sequences were trimmed and paired-end reads with sequence overlaps were merged into a single read that spans 
140 the full length of the original RNA fragment (dark orange; Fig 1E). The resulting single reads were

141 aligned to the Drosophila reference genome. The nucleotide at the 3' end of each RNA fragment was

142 identified and its genomic position recorded (asterisk; Fig 1E).

143 Two to three NET-seq libraries were independently prepared from early and late embryos using

144 S5P antibody; three additional libraries were prepared from late embryos using S2P antibody. Each

145 library was sequenced to a high coverage with a read length of 150 bp (Supplemental Fig S1A; see the

146 methods section for more detail). Experimental reproducibility was demonstrated by strong agreement

147 of uniquely aligned read density between biological replicates prepared with antibodies raised against

148 the CTD phosphorylated on either serine 5 (S5P) or serine 2 (S2P) positions (Supplemental Fig S1 B-

149 E). This suggests that both S5P and S2P antibodies recognize the CTD of elongating Pol II in 150 Drosophila embryos. 
A

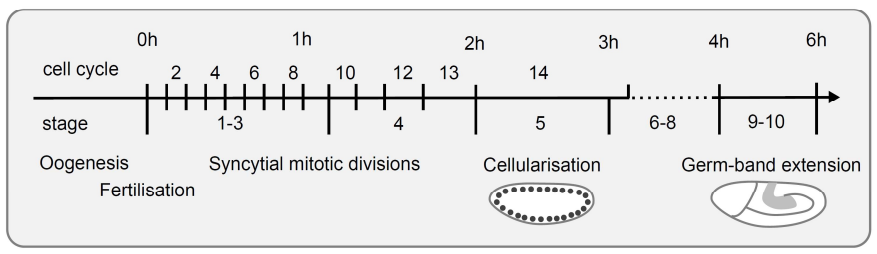

B

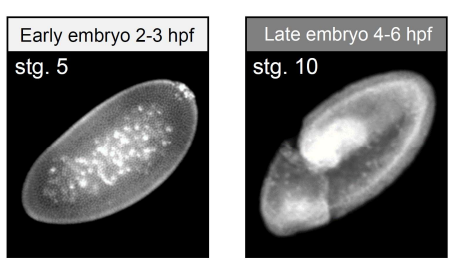

C

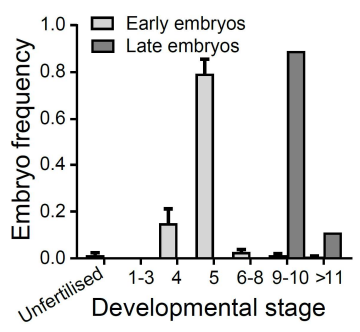

D

$\mathrm{E}$
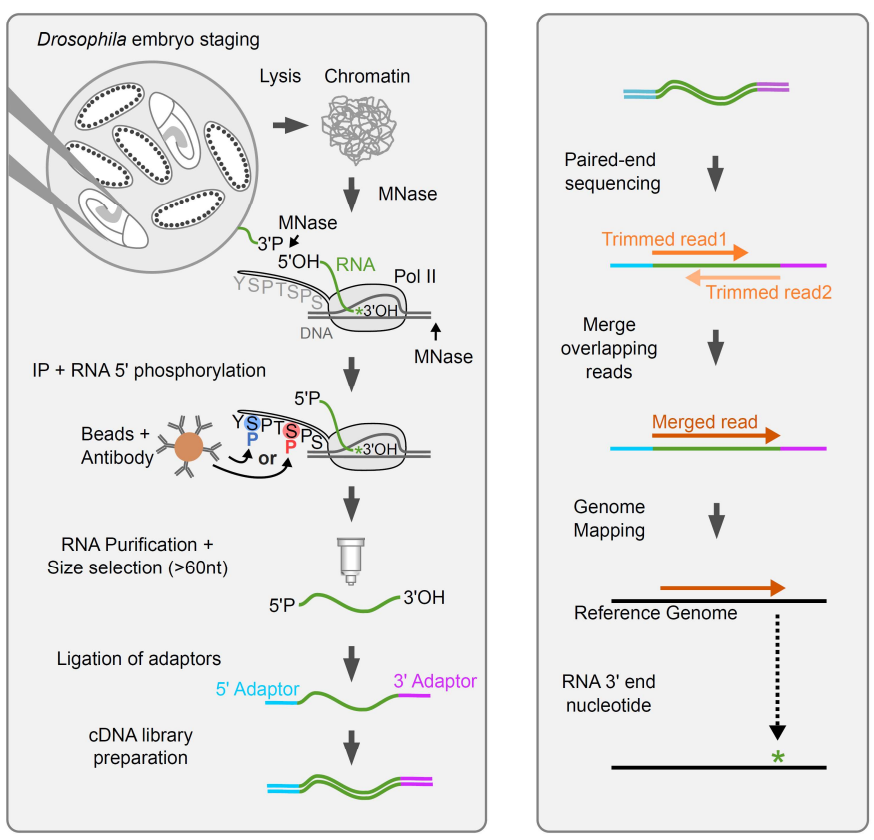

Figure 1. Native elongating transcript sequencing in Drosophila embryos.

(A) Timeline of Drosophila early embryonic development. (B) Representative images (stained for DNA) of embryos in mitotic cycle 14 (stage 5) and late germ-band expansion (stage 10). (C) The graph depicts the developmental stage of embryos sorted into the "early" and "late" groups. (D) Outline of the $d$ NET-seq experimental protocol. (E) Outline of $d$ NET-seq data analysis.

162 NET-seq captures not only the final (3' OH end) nucleotide of nascent RNA but also the 3' OH end of 
2017). Notably, in humans, mNET-seq of Pol II phosphorylated on CTD serine 5 detected splicing intermediates formed by cleavage at the 5 ' splice site after the first splicing reaction. The presence of such intermediates manifests as an enrichment of reads whose 3' ends map precisely to the last nucleotide of an exon. In both early and late Drosophila embryos, we indeed observed large peaks of $d$ NET-seq/S5P reads mapping to the last nucleotide of spliced exons, as shown for the $k u k$ gene (asterisk; Fig 2A). We also detected $d$ NET-seq/S5P peaks at the last nucleotide of introns, as shown for the eEFlalphal gene (asterisk; Fig 2B); enrichment for these reads results from coimmunoprecipitation of released intron lariats after completion of the splicing reaction (Fig 2B). In addition, we observed reads corresponding to mature snRNAs engaged in co-transcriptional spliceosome assembly, suggesting that $d$ NET-seq was capturing the free 3' $\mathrm{OH}$ ends of the snRNAs.

(Fig 2C). We found prominent peaks at the end of spliceosomal U1, U2, U4 and U5 snRNAs. As expected, no peak was detected mapping to the end of the U3 snRNA, which is involved in the processing of pre-rRNA synthesized by Pol I. Noteworthy, we observed an accumulation of $d$ NET-seq signal at the end of U6 snRNA (Supplemental Fig S2A), contrasting with a lack of peak observed in mammalian cells (Nojima et al. 2018). This is consistent with the finding that most mammalian U6 snRNAs contain a 2', 3'-cyclic phosphate terminal group at the 3' end, whereas U6 3' ends in Drosophila cells consist of either a cyclic 2',3'-phosphate, a 3'-phosphate or a 2',3'-hydroxyl group (Lund and Dahlberg 1992). an algorithm that finds nucleotides where the NET-seq read density is at least three standard deviations above the transcript mean in a local region defined by 100 bp upstream and downstream (Churchman and Weissman 2011; Prudêncio et al. 2020). Upon analysing replicates of $d$ NET-seq/S5P and $d$ NETseq/S2P libraries, we identified over 10,000 exons showing splicing intermediate peaks (Fig 2D). As peaks were more frequently detected on exons of genes with higher read density (Supplemental Fig S2B), we classified the exons into four groups (quartiles) based on the $d$ NET-seq read density of the corresponding gene and restricted the analysis to exons in the fourth quartile, i.e., from genes with the highest read density. The results show that splicing intermediate peaks are detected in approximately 
(Fig 2E). We then used the same methodology and the same set of genes to detect peaks at the last

A
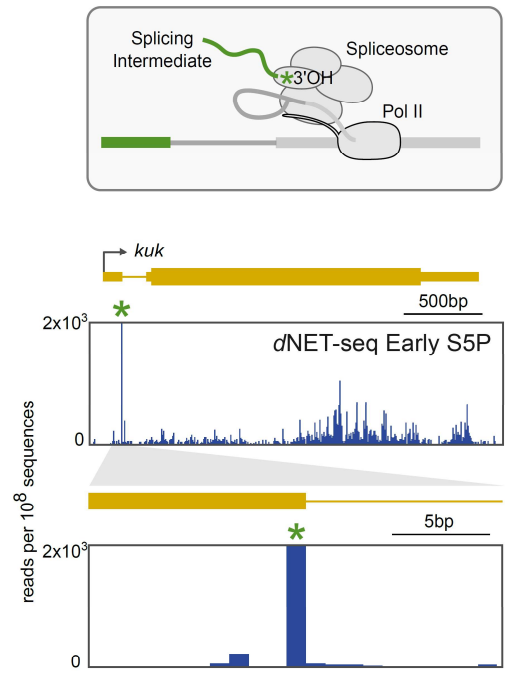

$\mathrm{D}$

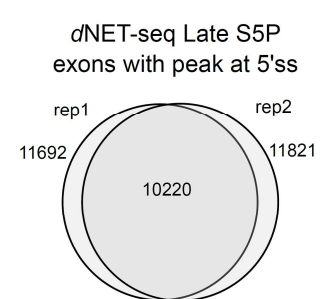

B
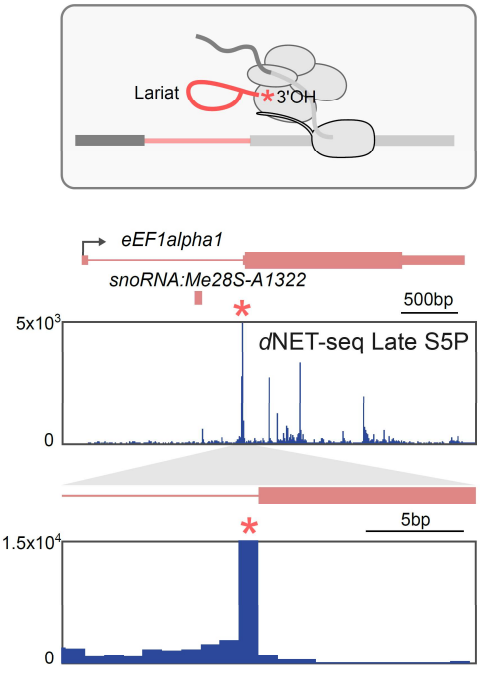

$\mathrm{E}$
C
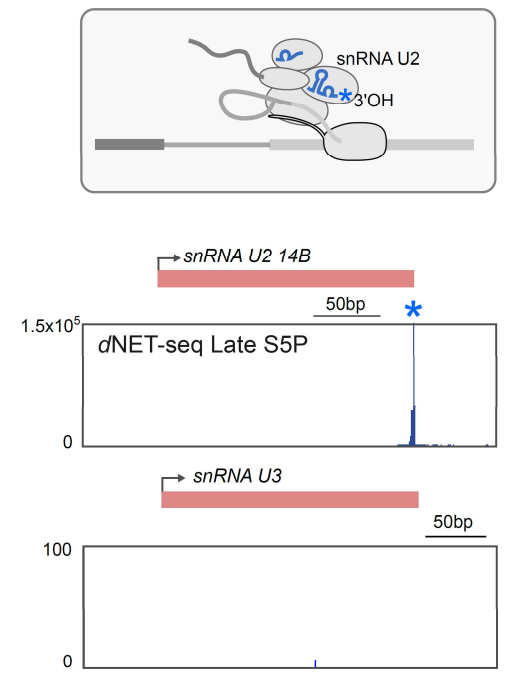

$\mathrm{F}$

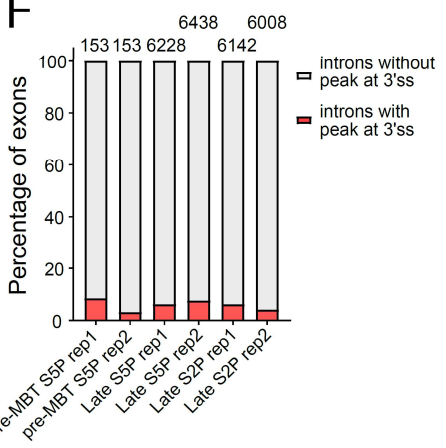

Figure 2. $d$ NET-seq captures splicing intermediates and spliceosomal snRNAs. (A-C) $d$ NETseq/S5P profiles over the indicated genes in late embryos. The diagrams outline the 3' $\mathrm{OH}$ ends generated by co-transcriptional cleavage at the 5' splice site (green, A), the 3' splice site (red, B) and the free 3' $\mathrm{OH}$ end of spliceosomal snRNAs (blue, C). Arrows indicate the direction of transcription. 
Exons are represented by boxes. Thinner boxes represent UTRs. Introns are represented by lines connecting the exons. Asterisks denote the peak at the end of the exon $(\mathbf{A})$, the end of the intron $(\mathbf{B})$, and the end of the U2 snRNA gene (C). (D) Comparison (Venn diagrams) of exons with a splicing intermediate peak detected in biological replicates of $d$ NET-seq/S5P and $d$ NET-seq/S2P libraries. (E, F) Frequency of peaks corresponding to splicing intermediates $(\mathbf{E})$ and released intron lariats $(\mathbf{F})$ in preMBT genes and genes expressed in late embryos. Only genes with the highest read density (fourth quartile) were considered.

\section{dNET-seq specifically captures nascent RNA.}

To validate that we were detecting nascent transcription in Drosophila embryos, we analysed maternal mRNAs that are transcribed during oogenesis and loaded into the egg (Fig 3A). As expected, maternal transcripts such as bicoid (Fig 3B), nanos (Supplemental Fig S3A), gurken (Supplemental Fig S3B) and Rab32 (Supplemental Fig S3C) were detected by RNA-seq in embryos collected 2-3 hours after fertilization, but $d$ NET-seq signal over these genes was negligible. However, robust $d$ NET-seq signal was found at the pumilio gene (Supplemental Fig S3D). Expression of this gene was considered to be exclusively maternal based on RNA-seq (Lott et al. 2011) and Pol II ChIP-seq data (Chen et al. 2013), but a GRO-seq study detected pumilio nascent transcripts in embryos collected 2-2.5 hours postfertilisation (Saunders et al. 2013). The detection of pumilio RNA in early embryos by GRO-seq and $d$ NET-seq highlights the sensitivity of these two techniques in capturing low-level nascent transcripts. The stage at which the embryo switches from relying on maternally deposited mRNAs and proteins to undergoing its own transcription is termed the mid-blastula transition (MBT). However, a few genes (known as pre-MBT genes) become transcriptionally active before MBT (Kwasnieski et al. 2019). Strong $d$ NET-seq signal was detected in early embryos over the bodies of pre-MBT genes such as snail (Fig 3C), fushi tarazu (Supplemental Fig S3E) and odd skipped (Supplemental Fig S3F). Altogether, we examined 117 previously identified pre-MBT genes and 35 maternal mRNAs (Fig 3D and Supplemental Fig S3G).

A characteristic feature of pre-MBT genes is that they are short in length with few if any introns (De Renzis et al. 2007; Ali-Murthy et al. 2013; Kwasnieski et al. 2019; Heyn et al. 2014). In contrast, most genes expressed in late embryos contain multiple introns, as shown for Akap200 (Fig 3E), His3.3A

(Supplemental Fig S3H) and twinstar (tsr) (Supplemental Fig S3I). Analysis of $d$ NET-seq datasets 
from late embryos revealed widespread signal on introns and regions downstream of the polyadenylation site, further confirming that the technique is capturing nascent transcripts.

To conclude, robust $d$ NET-seq signal was recovered from zygotically but not from maternally transcriptome.

dNET-seq reveals transcriptional readthrough associated with the presence of overlapping antisense genes.

Having confirmed that $d$ NET-seq was capturing nascent RNA, we next investigated the distribution of

Supplemental Fig S3) do not show the characteristic higher read density near the promoter, as previously described in mammalian cells (Nojima et al. 2015; Mayer et al. 2015). This is most likely because Pol II typically pauses $\sim 30-60$ bp downstream of the transcription start site (TSS) (Kwak et al. 2013) and in our $d$ NET-seq approach we enrich for RNAs longer than $60 \mathrm{nt}$ (Supplemental Fig S1F); thus, we only record the position of polymerases that have transcribed at least 60 bp past the TSS.

Fig S3H) and tsr (Supplemental Fig S3I), have considerable levels of $d$ NET-seq/S5P signal after the TES, indicative that in these cases, Pol II continues to transcribe after cleavage and polyadenylation. We noted that the 3' UTR of these three genes is overlapped by another gene (gurken, Nepl3, and IntSI, respectively), which is transcribed in the opposite direction. We therefore asked whether transcriptional readthrough is related to the presence of an overlapping convergent gene. In order to identify all the genes that are transcriptionally active in late embryos, we used a strategy adapted from GRO-seq analysis (Core et al. 2008) that relies on read density in gene desert regions as background reference for absence of transcription. Very large intergenic regions (gene deserts) were divided into 50kb windows, and read densities were calculated by dividing read counts in each window by the window length in bp 
percentile of read density for all intergenic regions analysed were considered to be transcriptionally active (Supplemental Fig S4B). We identified approximately 7 thousand active genes, and similar results were obtained from $d$ NET-seq/S5P and $d$ NET-seq/S2P datasets (Supplemental Fig S4C). This set of active genes includes over $85 \%$ of the 3500 genes previously identified as actively transcribed after the mid-blastula transition (MBT) based on ChIP-seq experiments (Chen et al. 2013). Next, we divided the genes transcribed in late embryos into two groups, depending on whether their 3' UTR was or was not overlapped by another convergent gene. The metagene analysis shows that when averaged across all transcribed genes, the $d$ NET-seq/S5P signal is abruptly reduced at the TES (Fig 3G). This is expected, as when Pol II reaches this site, the nascent transcript is cleaved and polyadenylated and therefore there is no RNA to be sequenced. The presence of nascent transcripts after the TES indicates that Pol II fails to terminate transcription after cleavage and polyadenylation. Unexpectedly, this is mainly observed on genes that have the 3' UTR overlapped by an antisense gene (Fig 3G). Similar results were observed after metagene analysis of $d$ NET-seq/S2P datasets (Fig 3H), confirming an association between transcriptional readthrough and genomic architecture at the TES. We then asked whether transcriptional readthrough depends on the transcriptional activity of the overlapping antisense (convergent) gene. Using the methodology described above to identify transcribed genes, we found that the vast majority $(>80 \%)$ of overlapping convergent genes were transcriptionally active and only 219 genes were silent. The metagene analysis shown in Fig 3I clearly indicates that transcription of the convergent overlapping gene is not required for inefficient termination at the overlapped TES. and Supplemental Fig S4D, E). Most likely, in these genes Pol II releases both the nascent RNA and the template DNA within a few nucleotides from the polyadenylation site. Notably, for the majority of pre-MBT genes devoid of $d$ NET-seq signal past the TES (65), there is no other gene on either strand within a region of $500 \mathrm{bp}$ downstream of the TES (referred to henceforth as 'isolated genes'), as shown for tailless (tll) (Supplemental Fig S4D). Another 26 pre-MBT genes are embedded in larger genes, as shown for nullo, which is located within a long intron of the CG12541 gene (Supplemental Fig S4E).

292 A smaller group of pre-MBT genes (21) have neighbouring genes located on either strand within a region of 500 bp downstream of the TES, as shown for Elba2 (Supplemental Fig S4F). We further 
294 identified 5 pre-MBT genes that have the 3' UTR overlapped by an antisense convergent gene, as shown

295 for spook (spo) (Supplemental Fig S4G). Transcriptional readthrough was observed in these pre-MBT

296 genes (Supplemental Fig S4G). Importantly, the proportion of isolated genes is significantly higher

297 among pre-MBT genes (65/117 or $\sim 56 \%)$ than among late genes $(1251 / 7233$ or $\sim 17 \%$ of all

298 transcriptionally active genes for replicate $1 \mathrm{~S} 5 \mathrm{P})\left(P<2.2 * 10^{-16}\right.$, one-tailed binomial test). This

299 suggests that pre-MBT genes may be under selection to avoid overlaps with other genes, thereby

300 minimizing transcriptional readthrough. Note, however, that we cannot exclude the possibility that in

301 some cases, a lack of NET-seq signal past the TES could be due to loss of CTD phosphorylation at this

302 position.

In conclusion, $d$ NET-seq reveals a strong correlation between inefficient transcription

304 termination at the cleavage and polyadenylation site, and presence of an antisense convergent gene overlapping the TES. The vast majority of genes that start to be transcribed before the mid-blastula transition, when the mitotic cycles are still very short, do not have the TES overlapped by other genes and lack $d$ NET-seq signal past the TES. In contrast, many genes that start to be transcribed after the mid-blastula transition, when the mitotic cycles become much longer, show transcriptional readthrough and are overlapped by an antisense convergent gene. 
bioRxiv preprint doi: https://doi.org/10.1101/2020 11.05.367888; this version posted July 25, 2021. The copyright holder for this preprint (which was not certified by peer review) is the author/funder, who has granted bioRxiv a license to display the preprint in perpetuity. It is made available under aCC-BY-NC-ND 4.0 International license.

A

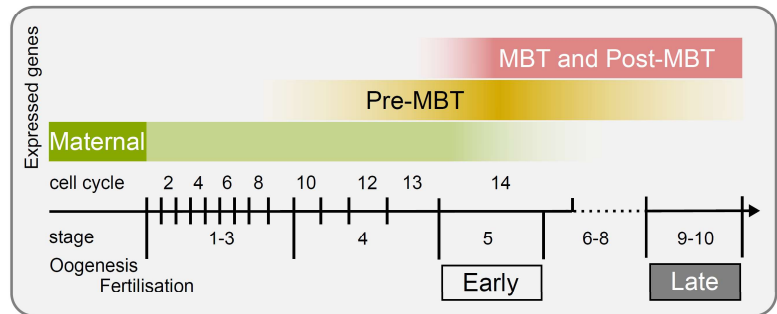

C

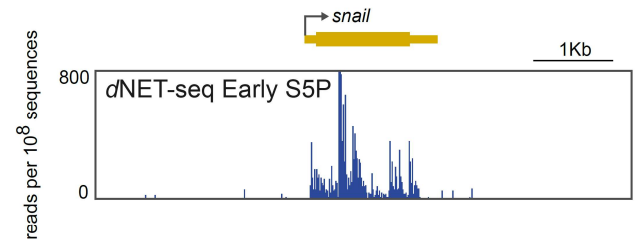

$\mathrm{E}$

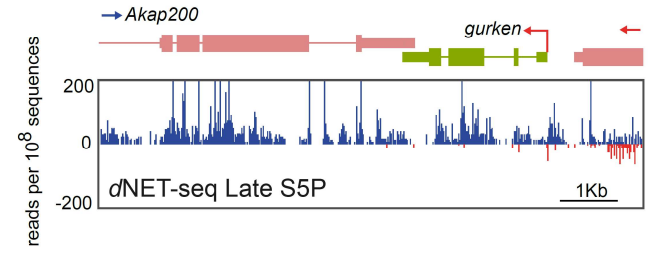

$\mathrm{F}$

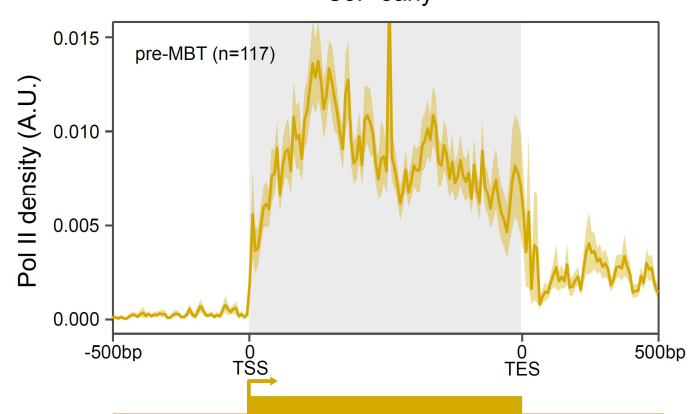

$\mathrm{H}$

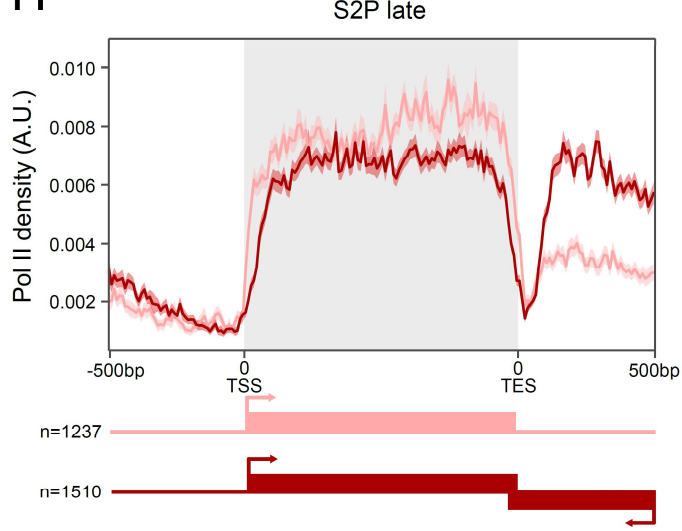

B

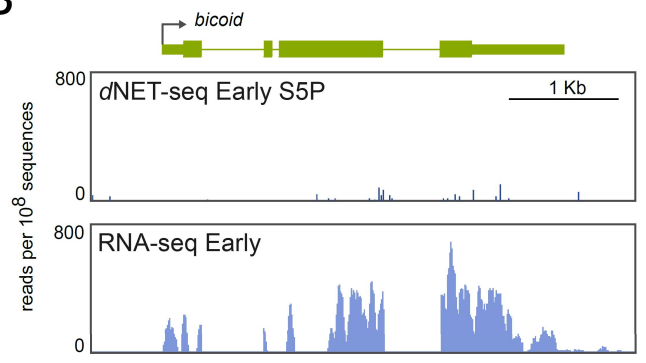

D

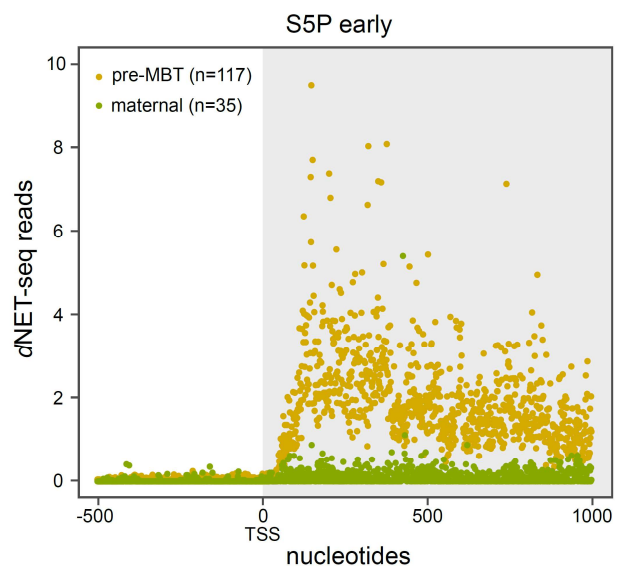

G

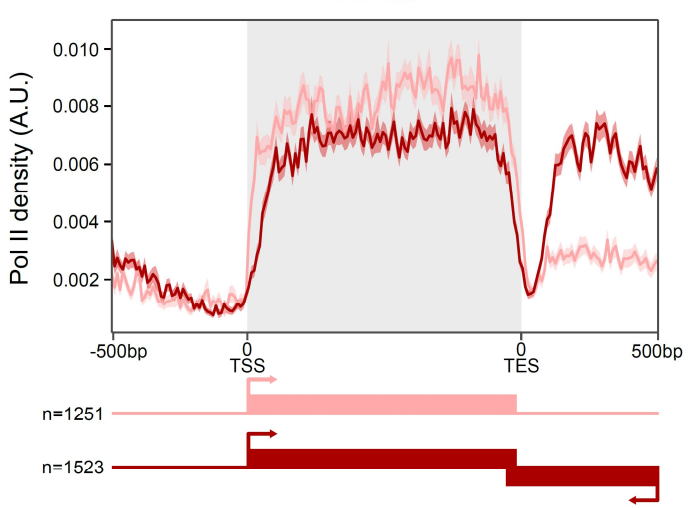

I

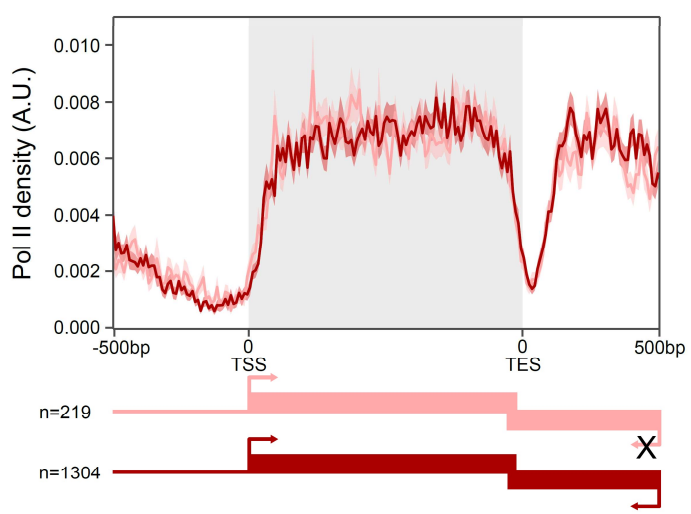


314 Figure 3. Transcription over gene bodies and beyond the TES.

315 (A) The diagram illustrates the temporal expression of maternal, pre-MBT, MBT, and post-MBT genes

\section{Analysis of $d$ NET-seq read density profiles.}

334 We next focused on the distribution of $d$ NET-seq reads on exons and introns. The number of nascent RNA reads whose 3' ends map at a particular genomic position is proportional to the number of Pol II molecules at that position. Thus, Pol II pause sites can be detected as local peaks in read density (Churchman and Weissman 2011; Larson et al. 2014). To search for $d$ NET-seq peaks representative of we were particularly interested in exploring the multiple peaks of dNET-seq/S5P signal observed on both exons and introns (Fig 4A), because co-transcriptional splicing has been linked to S5P in humans (Nojima et al. 2015).

A difficulty when performing the systematic identification of $d$ NET-seq peaks is that transcripts with higher initiation rates will contain more reads and thus peaks are more likely to be detected than in more lowly transcribed genes. To control for this confound, we developed a peak calling algorithm that detects regions where the local read density is significantly higher than expected by chance, given the over-all read density of the transcript (Fig 4A; see also the methods section). We emphasize that for 
looks for significant single-nucleotide positions (Churchman and Weissman 2011), whereas this new method detects higher read regions of variable length. at each position. Exons have a higher over-all peak density than introns (mean proportion of nucleotides in peaks $\sim 0.022 / \sim 0.015$ for replicate $1 /$ replicate 2 introns and $\sim 0.043 / \sim 0.034$ for replicate $1 /$ replicate 2 exons; $P<2.2 * 10^{-16}$ for both replicates; two-tailed Mann-Whitney $U$-test with the peak densities of individual introns/exons as data points), suggesting that the elongation rate is decreased over exons (Fig 4C). This is consistent with previous reports in mammalian (Mayer et al. 2015; Jonkers et al. 2014) and Drosophila cells (Kwak et al. 2013). In addition, peak density is sharply increased around the 5' splice site (Fig 4B). This could indicate Pol II pausing associated with splice site recognition. However, we cannot exclude that misaligned splicing intermediate reads are contributing to the observed increase in peak density around the 5' splice site.

The profile around the 3' splice site shows a higher peak density region just after the intronexon boundary (Fig 4B). A progressive increase in average exonic peak density is further observed starting roughly $60 \mathrm{nt}$ after the 3' splice site (Fig 4B), suggesting slower elongation or more frequent Pol II pausing in this region. Alternatively, such increases in peak density could correspond to regions where the Pol II CTD more frequently exhibits the phosphoisoform targeted by the antibodies. However, note that $d$ NETseq/Ser5 and $d$ NETseq/Ser2 lead to very similar peak density metaprofiles

\section{(Fig 4B; Supplemental Fig S7I-J).}

A further potential caveat is that nucleotide composition varies systematically across exons and introns. For example, exons tend to have a higher GC content than introns (Zhu et al. 2009). This could be problematic as NET-seq relies on MNase digestion of DNA and RNA to solubilize chromatin. MNase digestion of DNA is known to be sequence-biased, with most notably a preference for cleaving just 5' of an adenine (Dingwall et al. 1981; Hörz and Altenburger 1981; Gaffney et al. 2012). An analysis of the 5' ends of our reads revealed similar biases for MNase digestion of RNA (Fig 4D). This sequence preference could lead to artefactual variation in read density, with more reads being sampled

375 from transcripts and transcript regions whose nucleotide composition is more similar to MNase 376 digestion biases. To verify to what extent our results were affected by this confound, we performed a 
A
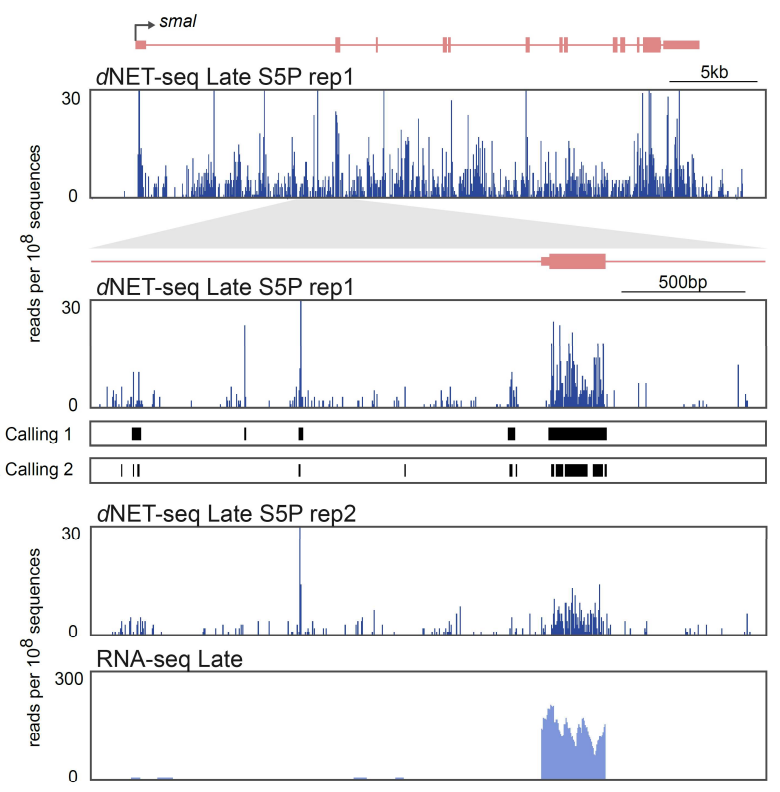

B

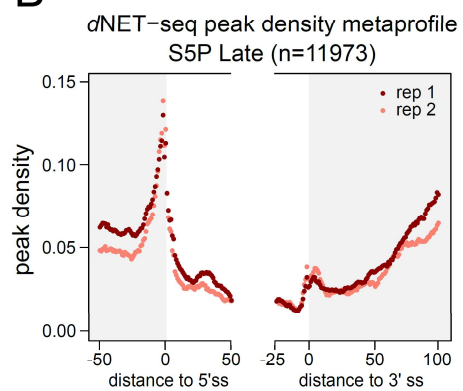

C

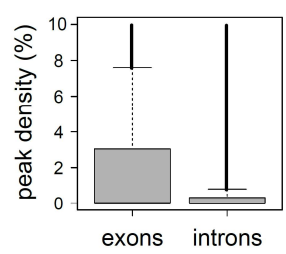

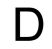

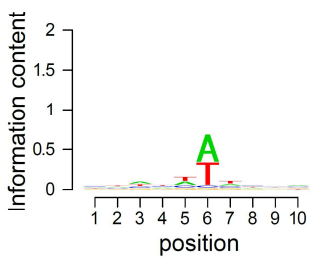

380

Figure 4. Analysis of $d$ NET-seq read density profiles.

(A) $d$ NET-seq/S5P and RNA-seq profiles over the post-MBT gene smoke alarm (smal). The read number is depicted at two magnification levels in two biological replicates. For replicate 1, the line "Peak Caller 1" shows peaks called using the "large peaks" setting, which is appropriate for detecting larger regions of putative Pol II pausing. The line "Peak Caller 2" shows peaks called using the "small peaks" setting, which provides higher spatial resolution and has been used for subsequent analyses. RNA-seq data for the same regions is also shown. (B) Metagene analysis of peak density estimated from $d$ NET-seq/S5P data from late embryos (replicate 1, N=12799). To calculate peak density for each position, we divided the number of introns that overlap with a peak at that position by the total number of introns. The last 50 nucleotides of exons, the first 50 nucleotides of introns, the last 25 nucleotides of introns, and the first 100 nucleotides of exons are shown. Only internal and fully coding exons from transcriptionally active genes that are at least 100 nucleotides long are shown. Exons shorter than 150 nucleotides contribute to both the exon end and start. Only introns that were at least $50 \mathrm{nt}$ long were considered. (C) Peak density in the exons and introns of transcriptionally active genes ( $d$ NET-seq/S5P, replicate 1). Peak density has been defined as the percentage of nucleotides within a given exon or intron that overlap with a significant peak. (D) Sequence logo of nucleotide frequencies within a 10-nt window around the 5' ends of NET-seq reads. The combined height of the bases at each position is proportional to the information content. Position 6 corresponds to the 5'-most nucleotide of the read. Putative internal priming reads, as well as reads mapping to the last nucleotide of exons or introns (possible splice intermediate and intron lariat reads) were ignored. 
405

406

407

408

409

410

411

412

413

414

415

416

417

418

419

420

421

422

423

424

425

426

427

428

429

430

431

Having shown that the spliceosome forms a complex with the elongating Pol II in Drosophila embryos, we asked when splicing takes place relative to transcription. We first looked at recursive splicing of long introns because this process involves the formation of inherently unstable intermediates that are more likely to be formed soon after the transcription of each intronic splice site (Pai et al. 2018). In recursive splicing, long introns are removed by sequential excision of adjacent sections involving separate splicing reactions, each producing a distinct lariat (Hatton et al. 1998). Recursively spliced intron segments are bounded at one or both ends by recursive sites or ratchet points (Burnette et al. 2005), which correspond to zero nucleotide exons consisting of juxtaposed 3' and 5' splice sites around a central AG|GT motif, where the vertical line represents the splice junction (Fig 5A).

To capture recursive splicing intermediates using $d$ NET-seq, it is essential to have a good coverage of reads corresponding to nascent transcripts and spanning the splice junctions. The total number of reads resulting from nascent RNA in each $d$ NET-seq dataset is depicted in Supplemental

Fig S1G. By merging the sequencing information of overlapped paired-end reads (Fig 1E), we were able to sequence on average $\sim 103$ nucleotides per nascent RNA (Supplemental Fig S1F and S1H). Focusing on previously identified Drosophila ratchet points (Joseph et al. 2018; Duff et al. 2015), we found $d$ NET-seq/S5P reads that span the junction between the canonical 5' splice site at the end of the exon and the first ratchet point (RP1) internal to the downstream intron, as shown for the second intron of the Megalin gene (Fig 5B). Reads spanning the subsequent intronic RPs were also observed (Fig 5B). Overall, we detected $d \mathrm{NET}$-seq/S5P and $d$ NET-seq/S2P spliced reads supporting most of the previously identified recursive splicing events (Fig 5C and Supplemental Fig S5A).

Analysis of $d$ NET-seq profiles around a RP reveals an enrichment of reads in a region located a few nucleotides downstream of the RP, as shown for RP2 in the first intron of the Tenascin major gene (Fig 5D). Noteworthy, most of these reads are already spliced to the previous RP (Fig 5D). A meta-analysis of $d$ NET-seq/S5P reads around 137 RPs confirms that many spliced reads can be observed just downstream of RPs (Fig 5E, F). Taken together, these results indicate that recursive splicing can occur soon after the transcription of intronic recursive sites and suggest the presence of paused Pol II downstream of these sites. 
A

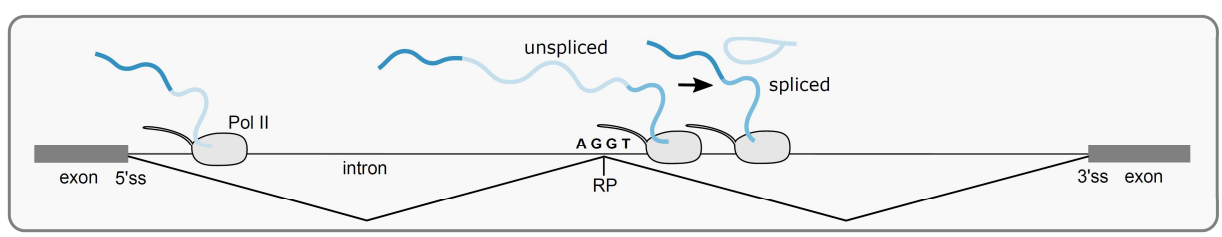

B

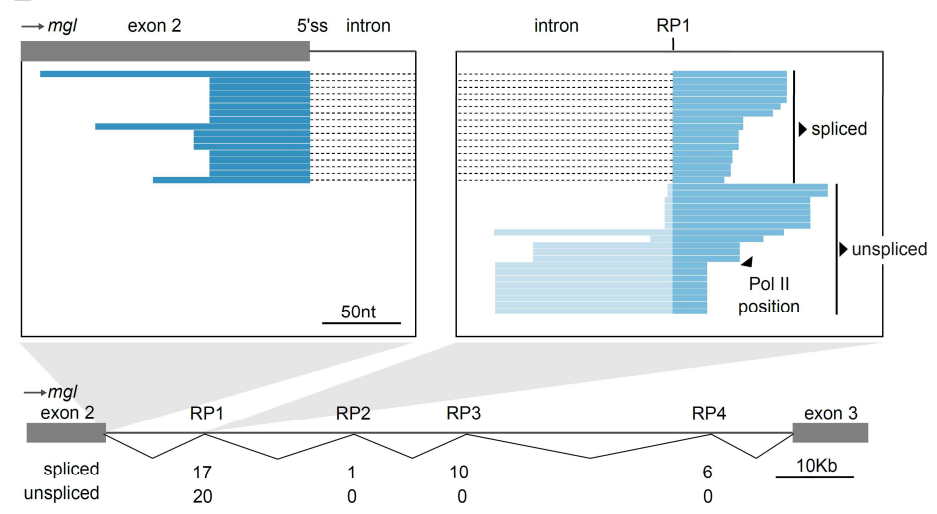

C

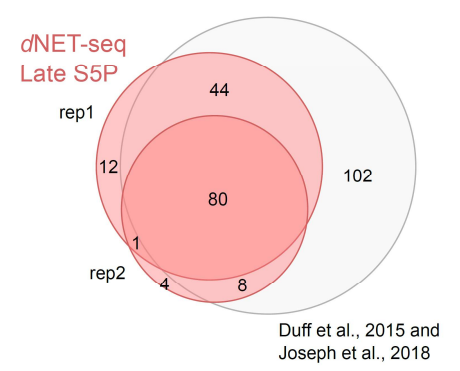

D

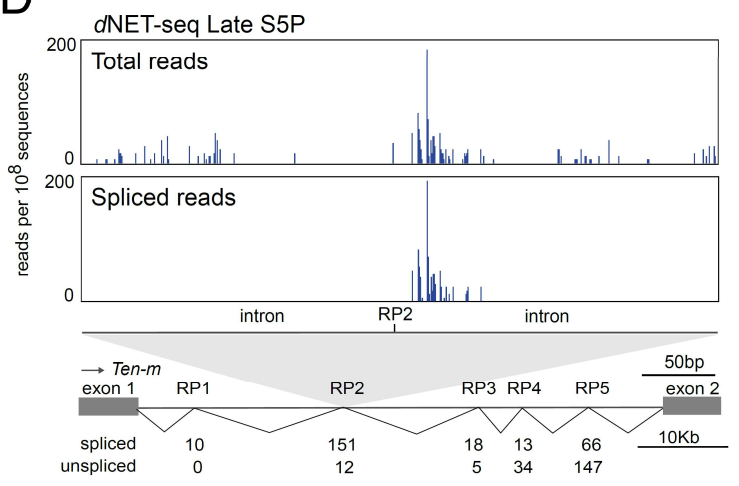

E

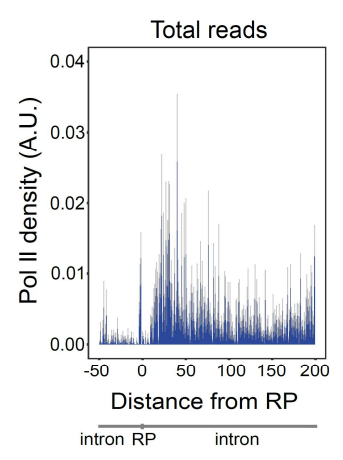

F

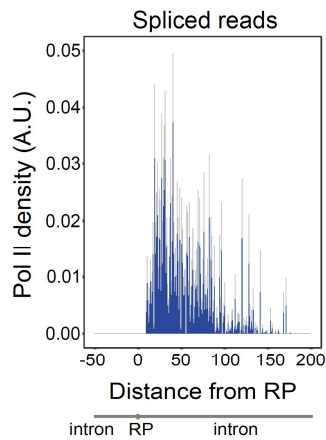

Figure 5. $d$ NET-seq captures recursive splicing intermediates.

(A) Schematic illustrating recursive splicing. A ratchet point (RP) with juxtaposed acceptor and donor splice site motifs is indicated. (B) Visualization of $d$ NET-seq/S5P reads that align to the second intron of the Megalin $(m g l)$ gene. Recursively spliced reads align to exon 2 (green) and the intron after RP1 (dark red). The number of spliced and unspliced reads at each RP in the intron is indicated. (C) Venn diagram comparing RPs identified in two $d$ NET-seq/S5P biological replicates and in previously reported studies (Joseph et al. 2018; Duff et al. 2015). (D) Number of $d$ NET-seq/S5P reads that have the 3' end mapped around RP2 in the first intron of Tenascin major (Ten-m) gene. The top panel depicts all reads, and the bottom panel depicts only reads that have been spliced to RP1. (E-F) Meta-analysis with single nucleotide resolution of normalized $d$ NET-seq/S5P reads around RPs $(n=137)$ using all reads $(\mathbf{E})$ or only reads spliced to the previous RP or exon $(\mathbf{F})$. 


\section{Splicing takes place as Pol II transcribes past the 3' splice site.}

450 Having established that $d$ NET-seq captures recursive splicing, we then asked whether $d$ NET-seq reads

451 spanning canonical exon-exon junctions were also detected (Fig 6A). To identify splicing events, we 452 considered all internal and fully-coding exons that are at least $100 \mathrm{nt}$ long in actively transcribed genes 453 in early and late embryos. For each splice junction, we counted how many reads had the 3' end mapped to the first $100 \mathrm{nt}$ of the exon. Only exons with at least 10 reads mapping to this region were considered (see the methods section for justification of the threshold). Then, the $d$ NET-seq splicing ratio (SR) was calculated by dividing the number of spliced reads by the sum of the number of spliced and unspliced reads (Fig 6A). Reads could be counted as spliced or unspliced if their 3' end mapped to within the first $100 \mathrm{nt}$ of the exon and their 5' end reached upstream of the 3' splice site, allowing to check whether the intron was still present. A robust agreement of estimated SR values was observed between biological replicates for pre-MBT genes (Supplemental Fig S6A) and genes expressed in late embryos (Fig 6B). $\mathrm{SR}$ values showed a bimodal distribution, with peaks at both extremes $(\mathrm{SR}=0$ and $\mathrm{SR}=1 ;$ Fig 6C and Supplemental Fig S6B), indicating that a subset of junctions were always spliced immediately after transcription $(\mathrm{SR}=1)$, while others remained unspliced $(\mathrm{SR}=0)$. Notably, differences in $\mathrm{SR}$ distribution were found between $d$ NET-seq/S5P and $d$ NET-seq/S2P datasets (Fig 6C, D and $<2.2 * 10^{-16}$ from binomial regression, see Fig 6 legend for statistical details). In particular, junctions that were most frequently spliced (SR values close to 1) were predominantly captured by $d$ NET-seq/S5P (Fig 6C and Supplemental Fig S6B). This clearly points to a preferential association between cotranscriptional splicing and S5 phosphorylation of Pol II CTD, as previously proposed in human cells (Nojima et al. 2015, 2018). We therefore continued to focus solely on the S5P datasets in the remainder of our splicing analysis.

We observed that only $\sim 5 \%$ of splice junctions in pre-MBT genes were devoid of S5P reads spanning ligated exons and thus presented an SR of $0(\sim 5.10 \%$ replicate $1 / \sim 4.35 \%$ replicate 2$)$, whereas in genes expressed in late embryos this proportion was $\sim 20 \%(\sim 19.72 \%$ replicate $1 / \sim 18.55 \%$ replicate

475 2) (Fig 6E; two-tailed binomial test for difference between late and pre-MBT, $\mathrm{P} \sim 6.141 * 10^{-5} / 9.44 *$ $10^{-4}$ (replicate $1 /$ replicate 2)). However, this difference between pre-MBT genes and genes expressed 
in late embryos disappeared once the higher read density of pre-MBT genes had been controlled for

$478 \quad($ Fig 6E).

Thus, in most cases, Drosophila co-transcriptional splicing can occur when Pol II is still

transcribing the downstream exon, implying an intron definition mechanism as previously proposed for have relatively long exons separated by short introns - a gene architecture suggested to be conducive to intron definition. Very long introns flanked by short exons have instead been associated with exon definition (under which the downstream exon needs to be fully transcribed before splicing can take place) (Keren et al. 2010). A switch to exon definition was proposed once the size of the intron surpasses $\sim 200 \mathrm{nt}$ (Fox-Walsh et al. 2005). It is unclear, however, whether the choice between exon and intron definition is dependent on the absolute sizes of exons and introns, or rather the ratio of intron to exon size.

We found no relationship between the $d$ NET-seq splicing ratio and exon length (Supplemental

Fig S6D, $\mathbf{H}$ for replicates 1 and 2; see Supplementary Table 1 for details on statistical significance and sample sizes for all of the gene architecture parameters discussed here). Regarding intron size, we found that introns with $\mathrm{SR}=0$ (and thus no evidence for intron definition) were, on average, indeed larger than other introns (Fig 6F and Supplemental Fig S6G). However, more careful examination revealed a more complex picture, with a lower proportion of introns with $\mathrm{SR}=0$ both for introns of intermediate size ( $\sim 55-100 \mathrm{nt}$, which corresponds to $\sim 55 \%$ of the introns studied) and for very large introns (>1000 nt) (Fig 6F). These intron sizes may thus be optimal for fast splicing. We also uncovered a lower exon to intron length ratio for introns with $\mathrm{SR}=0$ than for others (Supplemental Fig S6E, I; note that the difference was significant for replicate 2 and near-significant for replicate 1).

Taken together, these results suggest that although fast splicing (implying intron definition) is indeed skewed towards small introns flanked by large exons, there is also frequent and efficient intron definition for large introns. Our results are inconsistent with a threshold model, where splicing would systematically switch to exon definition after a given intron size is reached. parameters. Firstly, as the GC content in exons and introns decreases, the proportion of introns with SR 
$505=1$ increases and the proportion with $\mathrm{SR}=0$ decreases, showing more efficient immediate splicing $(\mathbf{F i g}$

506 6G, H and Supplemental Fig S6K, L). A similar effect is observed as the ratio of the downstream exon

507 GC content to intron GC content increases (Fig 6I and Supplemental Fig S6M). Thus, the most

508 efficient immediate splicing is observed when the GC content is low in both exons and introns but

509 higher in exons than introns. Secondly, as 3' splice site strength increases, the proportion of introns

510 with SR $=0$ decreases (Fig 6J and Supplemental Fig S6N). We found no significant relationship

511 between SR and 5' splice site strength (Supplemental Fig S6F, J). However, we replicated previous

512 observations (Khodor et al. 2012) that transcripts with only a single intron tend to be spliced less

513 efficiently than multi-intron ones, with a higher proportion of SR = 0 introns (Supplemental Fig S6O,

514 P). Thirdly, similarly to previous reports (Khodor et al. 2011, 2012; Herzel et al. 2018), we uncovered

515 an effect of exon rank, whereby exons that are more central appear to be spliced more efficiently (Fig

516 6K and Supplemental Fig S6Q). Finally, in light of recent results in mouse linking transcriptional

517 readthrough and inefficient splicing (Reimer et al. 2021), we asked whether such a relationship could

518 be detected in our data. We found no significant difference in levels of readthrough between genes with

519 high or low mean SR (Supplemental Fig S6R, S).

520 In conclusion, $d$ NET-seq reveals that splicing can occur immediately as Pol II transcribes past

521 the 3' splice site, yet many nascent transcripts remain unspliced. As expected, we found a relationship

522 between the proportion of spliced reads and intron size. However, immediate splicing was observed at all intron sizes. 
A

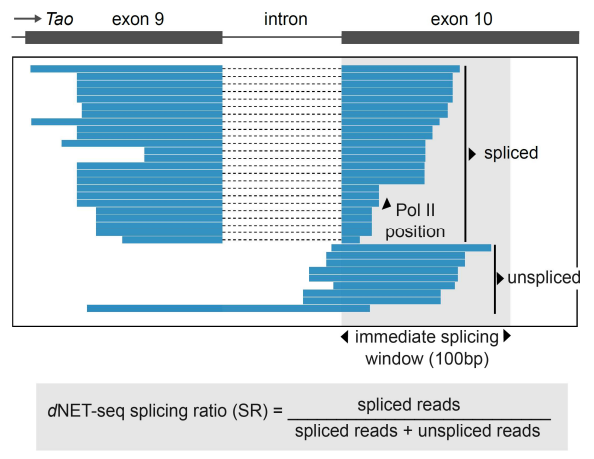

B

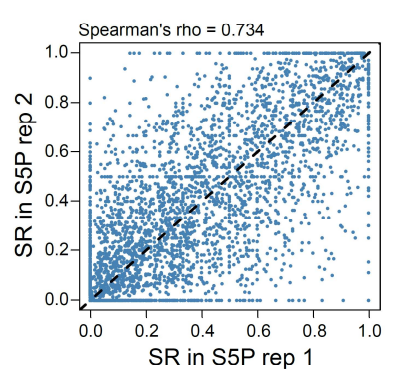

C

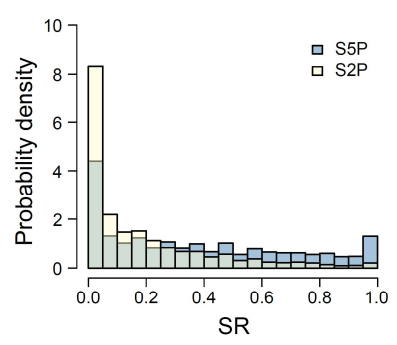

$\mathrm{D}$

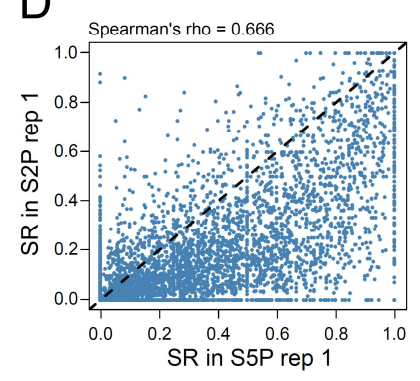

G

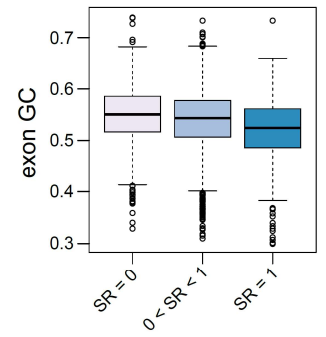

$\mathrm{J}$

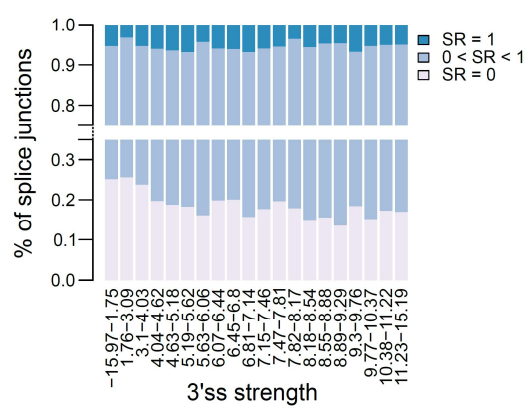

E

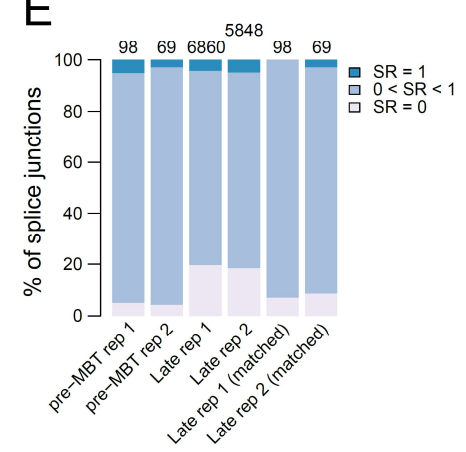

$\mathrm{H}$

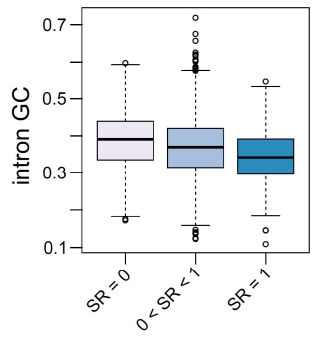

$\mathrm{K}$

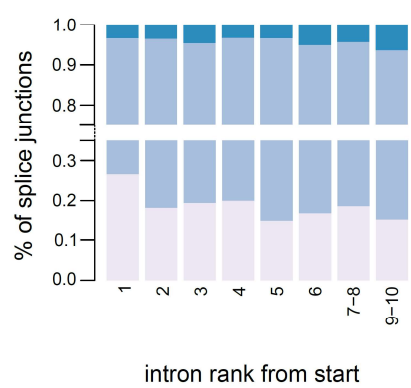

$\mathrm{F}$
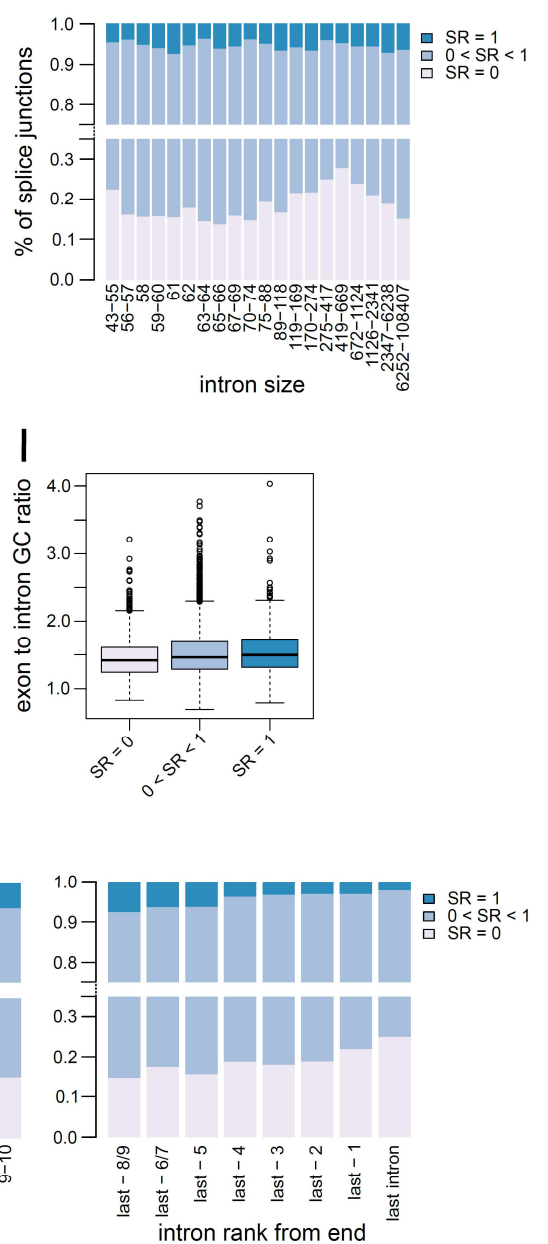

Figure 6. $d$ NET-seq reveals immediate splicing at all intron sizes.

(A) Visualization of $d$ NET-seq/S5P reads that align to exon 10 of the Tao gene. For (B-J), unless otherwise specified, only introns from transcriptionally active genes where the downstream exon is a fully coding internal exon at least $100 \mathrm{nt}$ long were included. In addition, enough spliced/unspliced reads had to end within the first 100 exonic nucleotides that obtaining a splicing ratio (SR) of 0 or 1 by chance alone was highly unlikely (see the methods section for details). For genes expressed in late embryos, this threshold was 10 reads for both replicates. For pre-MBT genes, it was 14 for replicate 1 and 9 for replicate 2. For S2P data, we used a threshold of 10 to enable better comparison with S5P. (B) 
535 (Spearman correlation, $\rho=\sim 0.734, P<2.2 * 10^{-16} ; \mathrm{N}=3708$ ). (C) Histogram of SR values for $d \mathrm{NET}$ $536 \mathrm{seq} / \mathrm{S} 5 \mathrm{P}(\mathrm{N}=5626)$ and $d \mathrm{NET}$-seq/S2P $(\mathrm{N}=6888)$. To test the significance of the difference between 537 S5P and S2P, a binomial regression with a logit link was performed without filtering by read number $(\mathrm{N}=12833$ for S5P; $\mathrm{N}=13229$ for $\mathrm{S} 2 \mathrm{P})$. The number of spliced and unspliced reads was specified as the dependent variable and the status of each data point as S5P or S2P was the sole predictor. The model predicted a splicing ratio of $\sim 0.369 / \sim 0.394$ for S5P replicate $1 / 2$ and of $0.162 / 0.269$ for S2P replicate 1/2. (D) SR values estimated in replicate 1 of $d$ NET-seq/S5P and $d$ NET-seq/S2P datasets from late embryos (Spearman correlation, $\rho=\sim 0.666, P<2.2 * 10-16 ; \mathrm{N}=4773$ ). (E) Proportion of splice junctions in pre-MBT genes and genes expressed in late embryos classified according to their SR values. As many pre-MBT genes are single-intron, last introns were exceptionally included in this analysis. To make the two columns on the right, we used a subset of the genes expressed in late embryos (post-MBT genes) that was as similar as possible to the pre-MBT set in read number. Concretely, to match each pre-MBT gene, we picked the post-MBT gene that had the most similar total count of spliced and unspliced reads, making sure that every post-MBT gene only appeared in the subset once. (F-K) Several parameters of gene architecture show a relationship with SR. For the sample sizes and statistical tests used, see Supplementary Table 1. Note that in (F, J-K), the bin ranges have been set so that intron numbers would be as equal as possible between bins.

\section{Immediate splicing associates with specific read density profiles.}

Analysis of individual $d$ NET-seq profiles around constitutive splice junctions with a high splicing ratio frequently revealed an enrichment of reads downstream of the 3' splice site, coincident with the appearance of spliced reads, as shown for the cno gene (Fig 7A). In contrast, profiles around a constitutive splice junction with $\mathrm{SR}=0$ had an accumulation of reads further along the exon, as shown for the ND-51 gene (Fig 7B). A clearly distinct type of profile was observed on skipped exons, on which very few reads were observed, as shown for the zip gene (Fig 7C). We next performed a meta-analysis of $d$ NET-seq peak densities over different exonic and intronic regions for introns with differing SR values. A difficulty of analysing the peak density profile around the 3' splice site is that reads mapping to the final nucleotide of the intron may represent intron lariats and thus be non-nascent. We removed reads mapping to this position prior to peak calling. However, through misalignment, intron lariat reads may also map to the few nucleotides around the 3' splice site and thus still affect the final meta-profile. In order to minimize the impact of such misalignment events, we excluded from the meta-analysis all introns with a read mapping to the final intronic nucleotide, as this is expected to also discard the introns most likely to contain misaligned intron lariat reads (Fig 7D, Supplemental Fig S7A). When such filtering is not done, a sharp peak is observed around the 3' splice site, notably when SR values are low (Supplemental Fig S7 B-C). It 
appears that intron lariat reads are primarily captured for introns with low splicing ratios (Fig $7 \mathbf{E}$ and

572 Supplemental Fig S7D). This contrasts with splicing intermediate reads from the end of the upstream

573 exon, which are associated to higher splicing ratios instead (Fig 7E and Supplemental Fig S7D).

The peak density profile downstream of the 3 ' splice site contains two primary regions of peak density located roughly 10-20 nucleotides after the 3' splice site. This peak is independent of the presence of putative intron lariat reads (Fig 7D, Supplemental Fig S7 A-C), and is thus likely to correspond to a genuine region of Pol II pausing or slow-down. However, it could also represent a region of preferential Ser5 phosphorylation, with no concomitant pausing. Noteworthy, pausing in this region has also been observed in studies that did not target specific Pol II phosphoisoforms (e.g. (Kwak et al. 2013)). Interestingly, this peak is more prominent when the SR is higher (Fig 7D, Supplemental

Fig S7 A-C, G-H), suggesting an association between immediate co-transcriptional splicing and increased Pol II pausing at the start of the exon.

7D, Supplemental Fig S7 A-C, G-H). We found no evidence that this increase in peak density could be a result of MNase digestion biases (Supplementary Methods). A further hypothesis is that this pattern could be reflecting a gradual slowdown of Pol II as it moves from an AT-rich intronic region to a GC-rich exonic one. The peak is indeed more prominent in introns where the ratio of exonic to intronic GC content is higher (Supplemental Fig S7 E-F). However, when SR=1, then even exons where this ratio is high do not display the peak (Supplemental Fig S7 E-F). Moreover, introns with higher SR tend to have higher exonic to intronic GC ratios (Fig 6I, Supplemental Fig S6M). Hence, the covariation with SR cannot be explained simply through the correlation between SR and GC content patterns. This suggests that there is an effect of the SR beyond any GC content effect. Potentially, this region could correspond to where delayed splicing starts (because these events have low immediate splicing ratios), and could represent splicing-associated Pol II pausing. peak densities (Supplemental Fig S7 G-H). Our results therefore cannot be explained through any 
biases introduced by the peak calling approach. The meta-profile obtained using $d$ NET-seq/S2P data was also qualitatively similar, although we did not divide the introns by SR given the rarity of reads

601 with high SR in S2P data (Supplemental Fig S7 I-J). We also attempted a separate meta-analysis of

602 skipped exons. These exons showed no accumulation of Pol II. However, the sample size was too small

603 for meaningful comparisons with included exons.

604

Finally, we asked how prevalent co-transcriptional splicing is in the developing Drosophila

605 embryo. As shown in Fig 2E, approximately $80 \%$ of exons in pre-MBT genes and genes expressed in

606 late embryos covered by a high density of NET-seq reads have a peak corresponding to the splicing

607 intermediate formed after cleavage at the 5' splice site but before exon-exon ligation. The majority of

608 these exons $(\sim 85 \%)$ were covered by $d$ NET-seq reads that span the junction to the downstream exon

609 either directly on nascent transcripts or indirectly on splicing intermediates formed by cleavage at the

6105 ' splice site of the downstream exon $(\mathbf{F i g} \mathbf{7 F}, \mathbf{H})$, confirming that they are co-transcriptionally spliced.

611 We then focused on those exons for which no splicing intermediate spike was detected by the peak

612 calling algorithm, as illustrated for the Doc3 gene (Fig 7G, I). Over $71 \%$ of these exons were also

613 covered by $d$ NET-seq reads that span the junction to the downstream exon either directly on nascent

614 transcripts or indirectly on splicing intermediates formed by cleavage at the 5 ' splice site of the

615 downstream exon (Fig 7I), arguing that even exons without a detectable splicing intermediate peak are

616 co-transcriptionally spliced. Altogether, our $d$ NET-seq results support co-transcriptional splicing for

617 over $95 \%$ of the analysed exons. 

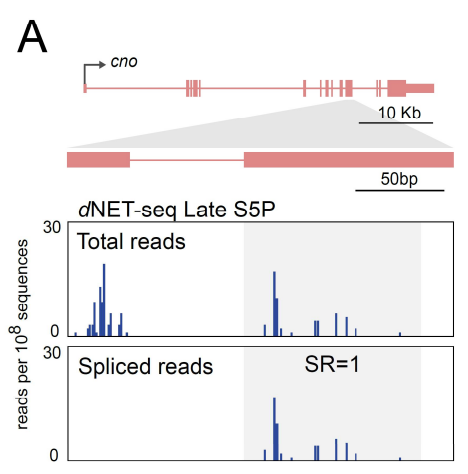

B
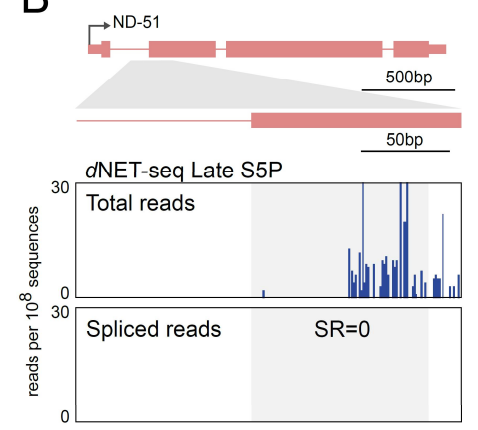

C
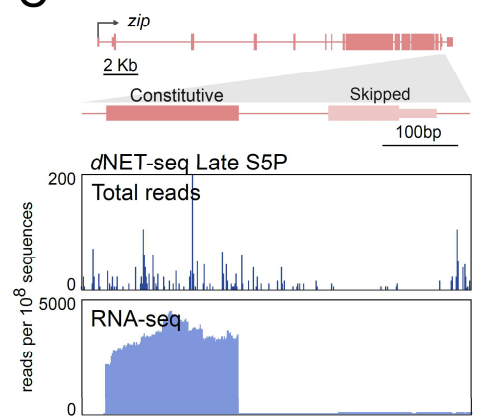

D

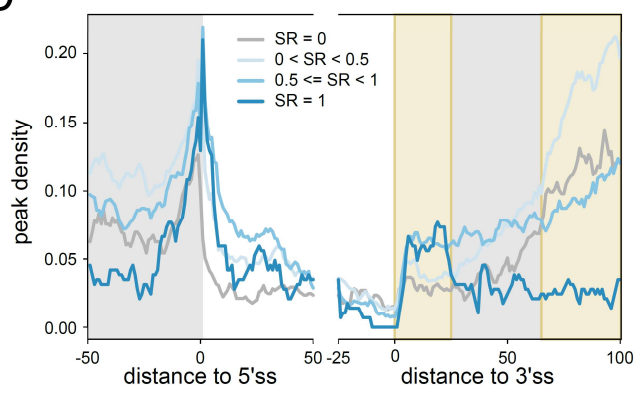

$\mathrm{F}$

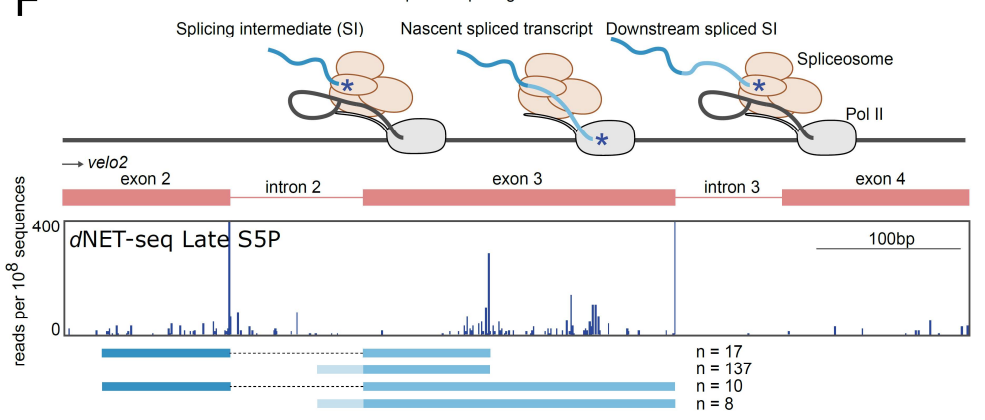

G

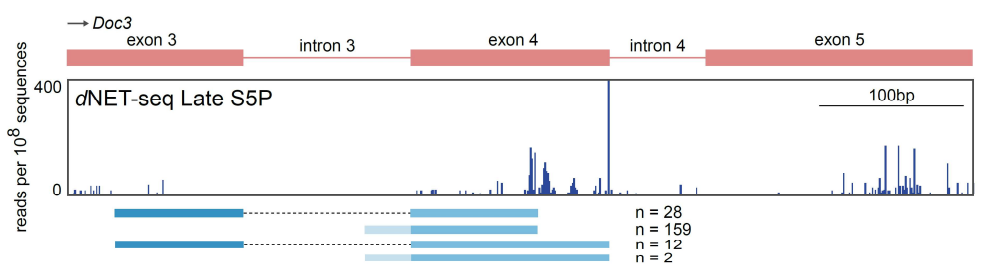

E

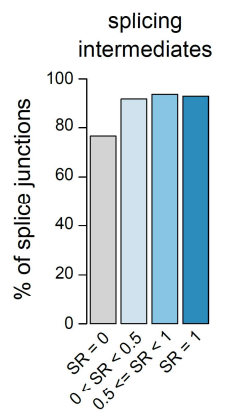

$\mathrm{H}$

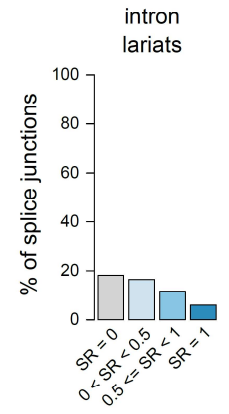

in

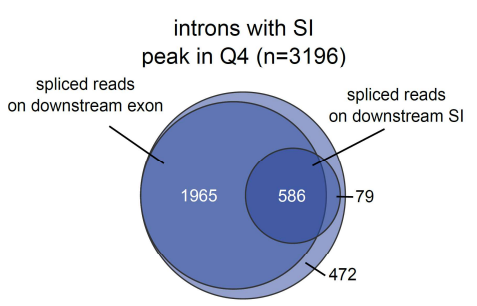

1

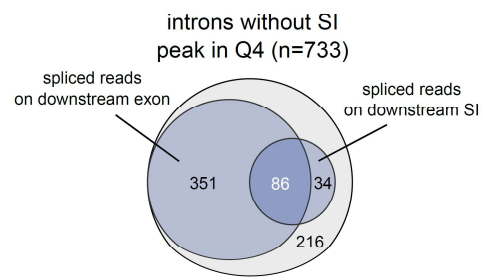

Figure 7. Immediate splicing associates with higher density of $\boldsymbol{d}$ NET-seq signal.

621 (A-C) $d$ NET-seq/S5P profiles surrounding the indicated exons in the post-MBT genes $c n o(\mathbf{A}), N D-51$

622 (B), and zip $(\mathbf{C})$. The top panels depict all reads. The bottom panels depict either the 3' end coordinate

623 of reads that span the splice junction (A, B), or the RNA-seq profile (C). (D) Metagene analysis of peak

624 density estimated from $d$ NET-seq/S5P datasets from late embryos (replicate 1) for different ranges of SR values. Peak density has been calculated as the proportion of introns that overlap with a peak at any given position. The last 50 nucleotides of exons, the first 50 nucleotides of introns, the last 25 nucleotides of introns, and the first 100 nucleotides of exons are shown. Only internal and fully coding exons from transcriptionally active genes that are at least 100 nucleotides long are shown $(\mathrm{N}=4783)$. In addition, at least $10 \mathrm{spliced} /$ unspliced reads had to end within the first 100 nucleotides of the exon. 
upstream exon (putative splicing intermediates) or to the final position of the intron (putative intron lariats) in $d$ NET-seq/S5P late replicate 1. (F, G) $d$ NET-seq/S5P profiles on the indicated regions of the velo 2 and Doc3 genes. Below, spliced reads are depicted. Asterisks denote the splicing intermediate peaks at the end of exons. $(\mathbf{H}, \mathbf{I})$ Venn diagrams showing how many junctions with or without a splicing intermediate peak are covered by spliced reads or have a downstream splicing intermediate covered by spliced reads.

\section{DISCUSSION}

In this study, we used $d$ NET-seq to map Pol II over the bodies of genes that become transcriptionally active in Drosophila embryos during the initial stages of development. The use of embryos allowed us to perform an important test to verify that the captured RNA is truly nascent. Indeed, early Drosophila embryos contain abundant mRNAs that are transcribed during oogenesis and loaded into the egg. In our analysis of early embryos, these maternal transcripts were readily detected by RNA-seq, but not by $d$ NET-seq.

Transcription in the Drosophila early embryo begins in mitotic cycle 8 for a few genes (Erickson and Cline 1993; Pritchard and Schubiger 1996) and then the number of active genes gradually increases until cycle 14 (Kwasnieski et al. 2019). Notably, the initial mitotic cycles have a duration of approximately 10 minutes, with cycle 13 taking about 21 minutes and cycle 14 lasting for at least 65 minutes (Ji et al. 2004). Thus, assuming an elongation rate of 2.4-3.0 kb per minute (Fukaya et al. 2017), there are significant constraints on the transcription of genes that are active before the end of cycle 13 (pre-MBT genes). Although pre-MBT genes are on average shorter than genes expressed at later stages of development (Artieri and Fraser 2014; Hoskins et al. 2011), longer genes are nevertheless transcribed before MBT. However, before cycle 14, transcriptional elongation of long genes is either prematurely terminated (Sandler et al. 2018) or aborted (Shermoen and O'Farrell 1991; Kwasnieski et al. 2019). It has also been reported that pre-MBT expression is associated with the generation of DNA damage due to stalling of DNA replication at transcriptionally engaged loci (Blythe and Wieschaus 2015).

Our $d$ NET-seq analysis revealed a novel feature of pre-MBT genes that most likely contributes to reduce conflicts between transcription and replication during the short interphases of early embryos. We found that in the majority of pre-MBT genes, no $d$ NET-seq signal is detected after the TES, whereas many of the genes that are only expressed after MBT show high $d$ NET-seq signal past the TES. We 
662 also observed that most pre-MBT genes are isolated from other transcriptional units, while the TES of 663 many post-MBT genes is overlapped by another gene transcribed in the opposite direction. Taken 664 together, these results suggest that Drosophila genome architecture may be under selection for efficient 665 transcription termination of pre-MBT genes. Our observations further reveal an unexpected link 666 between transcriptional readthrough and the presence of an overlapping convergent gene in the Drosophila genome. The finding that readthrough is detected even when the convergent overlapping gene is not transcribed is intriguing. One possibility is the induction of local conformational changes in chromatin and/or Pol II, Indeed, pioneer transcription factors can potentially regulate gene expression at later stages of development by inducing significant chromatin conformational changes during early embryonic development (Blythe and Wieschaus 2016; Schulz et al. 2015). Although such chromatin remodelling events can potentially influence transcriptional read-through, regardless of convergent gene transcription, further studies are needed to understand how transcription termination is regulated during early Drosophila development.

A classical view in the splicing field is that for some introns, the 5' and 3' splice sites are recognized directly by 'intron definition'. For other introns, spliceosome assembly starts with the recognition of the downstream exon, and only at a later stage a cross-intron complex is formed ('exon definition') (Robberson et al. 1990; Berget 1995; Sterner et al. 1996; Fox-Walsh et al. 2005). These alternative models are supported by in vitro evidence, as well as by in vivo reporter gene experiments (Robberson et al. 1990; Berget 1995; Fox-Walsh et al. 2005; De Conti et al. 2013). There is also indirect evidence, for example from analysis of exon-intron size patterns (Berget 1995). However, these models have never been directly tested in vivo at a transcriptome-wide level. It thus remains uncertain how intron definition and exon definition operate in vivo and what determines the choice between them. are necessarily intron-defined, as exon definition requires the presence of the 5' splice site of the downstream intron. Therefore, introns with an SR above 0 represent an experimentally-determined set of introns that are spliced via intron definition at least part of the time, allowing us to test long-held assumptions in the field. Notably, in vitro and reporter gene work suggested that Drosophila uses intron definition for introns smaller than $\sim 200-250$ nt and exon definition for larger introns (Fox-Walsh et al. 
2005). Other studies have suggested that the crucial factor is not just intron size but the relative size of

691 the intron compared to the flanking exons (Sterner et al. 1996). the proportion of introns with $\mathrm{SR}>0$, intron definition can be observed at all intron sizes. Our results are thus inconsistent with a simple "threshold" model, where splicing would systematically switch to exon definition at a particular intron size. Rather, we observe an increased proportion of introns with SR $>0$ within an optimal intron size range of $\sim 55-100 \mathrm{nt}$. The median intron size in our data set is 71 nt. It thus appears that intron definition is best optimized for introns of a "typical" size, and is less efficient when introns are unusually small or large. Intriguingly, however, for very large introns $(>1000$ $\mathrm{nt})$, the proportion with $\mathrm{SR}>0$ increases again, suggesting that specific mechanisms may have evolved for the intron-defined splicing of very large introns. The tendency for faster splicing kinetics with average intron sizes has also been reported using metabolic labelling in both Drosophila (Pai et al. 2017) and human cells (Windhager et al. 2012). 2008) could be crucial for the intron-defined splicing of such large introns. Indeed, introns with SR $>0$ where the upstream exon has one or more putative splicing intermediate reads tend to be larger than ones without splicing intermediate reads (one-tailed Mann-Whitney $U$-test, $\mathrm{W}=779623, \mathrm{P} \sim 0.003, \mathrm{~N}$ =4589; exon size and read number filtering has been applied like for the gene architecture analysis). similar relationship with GC content, with the highest proportion of introns with $\mathrm{SR}>0$ observed when both the exon and the intron were AT-rich but the exon had a higher GC content than the intron. We emphasize that the gene architecture parameters that we have investigated are not independent of eachother. For instance, exon GC content is correlated negatively with exon rank from start (Spearman's $\rho$ $\sim-0.098 ; \mathrm{P} \sim 1.196 * 10^{-12}$ ). Further work is thus needed to distinguish between causative and merely correlative factors. In addition, it must be determined how the GC content effect relates to patterns of nucleosome density (Gelfman et al. 2013). can be spliced through intron definition. . A caveat of our work is that introns that show no evidence 
718 for splicing completion during transcription of the downstream exon could still be spliced via intron

719 definition, if their splicing takes too long for it to be detected during the $100 \mathrm{nt}$ window studied.

720 Therefore, we do not know whether introns with a SR of 0 are exon-defined, intron-defined but spliced

721 when Pol II has elongated past the first 100 exonic nucleotides, or a mixture of both. Similarly, introns

722 with a splicing ratio between 0 and 1 could either always be intron-defined, or they could use either

723

724

725

726

727

728

729

730

731

732

733

734

735

736

737

738

739

740

741

742 exon or intron definition depending on the splicing event.

Taken together, our results show that splicing in Drosophila embryos can be completed very rapidly after transcription of the 3' splice site, as previously reported in other cellular models (Carrillo Oesterreich et al. 2016; Reimer et al. 2021; Martin et al. 2013). Furthermore, our findings are relevant to controversies regarding Pol II pausing in association with splicing. Indeed, splicing-dependent Pol II pausing near the 3' splice site was first reported in yeast (Alexander et al. 2010; Chathoth et al. 2014). Evidence for Pol II pausing at exon boundaries was further detected in mammalian cells using NETseq (Nojima et al. 2015; Mayer et al. 2015). Notably, NET-seq density profiles suggestive of Pol II pausing were observed with S5P antibodies but not with antibodies to unphosphorylated CTD (Sheridan et al. 2019; Nojima et al. 2015). In contrast, a recent PRO-seq analysis found no splicing-associated Pol II pausing (Reimer et al. 2021).

Finally, we emphasize that our analysis of nascent Drosophila transcripts associated with Pol II S5P CTD revealed highly heterogeneous splicing dynamics. Indeed, while only a minority of splice junctions were devoid of reads spanning ligated exons (corresponding to an SR of 0), many introns remained unspliced in our analysis. Is it relevant for cells whether any particular intron is rapidly excised after Pol II transcribes the 3' splice site or is left unspliced while the transcript elongates? Is this a stochastic process? What are the consequences for gene expression of fast versus delayed splicing? Addressing these questions will likely dependent on further advances in methodologies to study splicing in time and space at a transcriptome-wide level. 


\section{METHODS}

\section{Embryo collection} syrup). Three-day-old flies (counting from pupae eclosion) were fattened in culture medium supplemented with fresh yeast for 2 days. Embryos were collected into apple juice-agar plates supplemented with fresh yeast using appropriate cages containing approximately 200 flies each. To avoid female retention of older embryos, three pre-collections of 30min each were made before the first hours after egg-laying), adult females were allowed to lay eggs for 1 hour in apple juice-agar plates. Plates were subsequently collected and embryos were aged at $25^{\circ} \mathrm{C}$ for $90 \mathrm{~min}$. During the following 30 min, embryos were harvested from the plates, dechorionated in $50 \%$ bleach solution for $2 \mathrm{~min}$, and washed once in Phosphate Buffered Saline supplemented with 0.1\% Tween-20 (PBT) and twice in deionized water. In order to discard older embryos (stage 6 and older), manual staging of collected embryos was performed with the help of forceps and under a magnifier scope. Embryos were then resuspended in a solution containing $120 \mathrm{mM} \mathrm{NaCl}$ and $0.04 \%$ Triton, and washed twice with $120 \mathrm{mM}$

$\mathrm{NaCl}$ solution. At the end of the 3 hours collection, the solution was removed and embryos were frozen in liquid nitrogen and stored at $-80^{\circ} \mathrm{C}$. For the late stage (4-6 hours), eggs were laid for 2 hours and aged at $25^{\circ} \mathrm{C}$ for 3.5 hours. Embryo collection and processing was similar to the early stage embryos, but in this case, no manual staging was performed.

\section{Embryo DNA staining}

For each embryo collection, and after dechorionation, a representative embryo sample was collected from the total pool and fixed in a scintillation flask, using a solution containing 1 volume of 
$7714 \%$ formaldehyde in PBT and 4 volumes of heptane, for $20 \mathrm{~min}$ at 100rpm. The lower aqueous phase

772 solution was subsequently removed, $4 \mathrm{ml}$ of methanol was added and embryos were shaken vigorously

773 during 1 min. Embryos were then collected from the bottom of the scintillation flask, washed twice

774 with methanol, and frozen at $-20^{\circ} \mathrm{C}$ in methanol. To rehydrate the embryos, they were washed for 5

775 min each, with 3:1, 1:1, and 1:3 mix solutions of methanol:PBT. Embryos were subsequently washed

776 twice in PBT and incubated with 1:5000 Sytox green (Invitrogen), supplemented with 5 $\mu \mathrm{g} / \mathrm{ml}$ RNase

777 A (Sigma-Aldrich) in PBT for 15 min. After washing with PBT, embryos were mounted in fluorescence

778 mounting medium (Dako) and examined in a Zeiss AxioZoom V16 Fluorescence Stereo Microscope

779 for image acquisition and embryo staging. Images were processed using ImageJ software (NIH).

\section{$d$ NET-seq and library preparation}

782

The $d$ NET-seq protocol was adapted from mNET-seq (Nojima et al. 2016). Briefly, $300 \mathrm{ul}$ of frozen embryos was resuspended in 3.5ml of Buffer B1 (15mM HEPES-KOH, pH 7.6; 10mM KCl; suspension was again homogenised in the Dounce with $4 \mathrm{x}$ strokes and loaded without mixing on the top of buffer B2 (15mM HEPES-KOH, pH 7.6; 10mM KCl; 5mM MgCl2; 1mM DTT; 0.1mM EDTA; 0.8M Sucrose; $4 \mu \mathrm{g} / \mathrm{ml}$ Pepstatin; 10mM Sodium Metabisulfite; 0.5mM EGTA supplemented with complete EDTA free protease inhibitor (Roche) and PhosSTOP (Roche)). The suspension was centrifuged at $1310 \mathrm{~g}$ during $30 \mathrm{~min}$ at $4^{\circ} \mathrm{C}$, and the pellet was resuspended with $125 \mu \mathrm{l}$ of NUN1 buffer inhibitor (Roche) and PhoSTOP (Roche) was mixed with the nuclei and incubated on ice during 15 min preforming short vortex every $3 \mathrm{~min}$. Chromatin was then centrifuged at $10000 \mathrm{~g}$ for $10 \mathrm{~min}$ at $4^{\circ} \mathrm{C}$, 


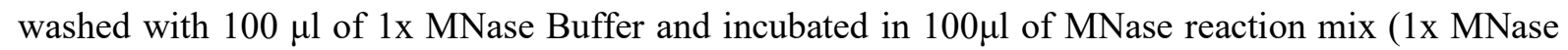

800 buffer and 30 gel unit/ $\mu \mathrm{l}$ MNase (New England Biolabs)) during 3 min at $37^{\circ} \mathrm{C}$ with mix at $1400 \mathrm{rpm}$.

801 The reaction was stopped with $10 \mu \mathrm{l}$ of $250 \mathrm{mM}$ EGTA, centrifuged at $10000 \mathrm{~g}$ for $5 \mathrm{~min}$ at $4^{\circ} \mathrm{C}$, and the

802 supernatant containing the solubilized chromatin was recovered. For the early embryos sample, $2 \mathrm{x}$

$803300 \mu 1$ of embryos were prepared in parallel and pooled together after the chromatin solubilization.

804 Immunoprecipitation of Pol II-RNA complexes was performed using 50 $\mu$ l of Protein G Dynabeads

805 (Thermo Fisher Scientific), pre-incubated over night with 5 $\mu$ g of the correspondent antibody: anti-Pol

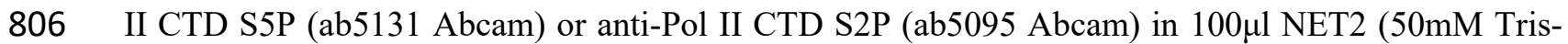

$807 \mathrm{HCl} \mathrm{pH} \mathrm{7.4;} 150 \mathrm{mM} \mathrm{NaCl}$ and $0.05 \% \mathrm{NP}-40$ ) and washed 3 times with NET2. Beads were incubated

808 with the solubilized chromatin in $1 \mathrm{ml}$ total volume of NET2 during 1 hour at $4^{\circ} \mathrm{C}$, washed 7 times with

$809500 \mu$ l of NET2 and once with $100 \mu \mathrm{l}$ of PNKT (1x PNK buffer and $0.1 \%$ tween) before incubation

810 during $6 \mathrm{~min}$ in $50 \mu \mathrm{l}$ of PNK reaction mix (1x PNKT, $1 \mathrm{mM}$ ATP and $0.05 \mathrm{U} / \mathrm{ml}$ T4 PNK 3'phosphatase

811 minus (NEB) in a thermomixer at $37^{\circ} \mathrm{C}$ and $1400 \mathrm{rpm}$. After washing the beads with NET2, long RNA

812 fragments were isolated using Quick-RNA MicroPrep (Zymo research): 300 $\mu$ of RNA Lysis Buffer in

$81333 \%$ EtOH was mixed to the beads by pipetting. Beads were discarded and the suspension was loaded

814 into a Zymo spin column that was centrifuged at $10000 \mathrm{~g}$ during $30 \mathrm{sec}$. The column was washed once

815 with $400 \mu 1$ RNA prep buffer and twice with $700 \mu 1$ and $400 \mu 1$ RNA wash buffer respectively. RNA was

816 then eluted in $15 \mu \mathrm{l}$ of DNase/RNase-Free water (zymo) and stored at $-80^{\circ} \mathrm{C} .100 \mathrm{ng}$ of RNA was used

817 to prepare each library, following the standard protocol of the Truseq small RNA library prep kit

818 (Illumina). After adapter ligation and reverse transcription, the libraries were PCR amplified using 16

819 PCR cycles and cDNA libraries were fractionated in the gel between 130 to $300 \mathrm{bp}$. The libraries were

820 sequenced using PE-150 on the Illumina HiSeq X platform by Novogene Co., Ltd.

821

822

823

824

825

826 


\section{QUANTIFICATION AND STATISTICAL ANALYSIS}

\section{$828 d$ NET-seq data processing}

829

830

Adapter sequences were removed from all $d$ NET-seq paired-end samples using Cutadapt

831 (version 1.18) (Martin 2011) with the following parameters: -a TGGAATTCTCGGGTGCCAAGG -A

GATCGTCGGACTGTAGAACTCTGAAC -m 10 -e 0.05 --match-read-wildcards -n 1. Paired-end read merging was performed using bbmerge.sh from BBMap (Bushnell et al. 2017) with the 'xloose'

834 parameter. Merged reads were then aligned to the Drosophila reference genome ( $d m 6$; Ensembl release

835 95) (Cunningham et al. 2019) using STAR (version 2.6.0b) (Dobin et al. 2013) with -chimSegmentMin

836 set to 20. Only uniquely mapped reads were considered, extracted using SAMtools (version 1.7) (Li et

837 al. 2009) with -q set to 255. Exceptionally, in Supplemental Fig S2A, HiSat2 was used, using the same

838 dm6 genome annotation file for genome indexing and using default parameters, with the --no-

839 discordant -no-mixed flags set. PCR internal priming events generated during library preparation were

840 removed using a custom Python script (Prudêncio et al. 2020) with the following parameters: -a TGG..

841 -s paired. To obtain single-nucleotide resolution, a custom Python script (Prudêncio et al. 2020) was

842 used to extract the $5^{\prime}$ end nucleotide of read 2 (after trimming) in each sequencing pair, with the

843 directionality indicated by read 1 (Fig 1E).

844

Publicly available RNA-seq datasets used

846

847

848

849

850

851

852

853

854

Publicly available Drosophila embryonic transcriptome sequencing data (Poly(A) RNA-seq), performed in developmental stages similar to the dNET-seq early and late samples, were used in this study. RNA-seq datasets corresponding to cycle 14B (Lott et al. 2011) were obtained from the Gene Expression Omnibus (GEO) (samples GSM618409, GSM618410, GSM618421 and GSM618422 from dataset GSE25180). RNA-seq datasets from 4-6h old embryos were obtained from modENCODE project PRJNA75285 (Graveley et al. 2011) (accessions SRR023696, SRR023746, SRR023836, SRR035220, SRR023669, SRR035405, SRR035406, SRR024014 and SRR023539). 
RNA-seq data processing

856

Adapters were removed from all datasets using Trim Galore (version 0.4.4)

858 (http://www.bioinformatics.babraham.ac.uk/projects/trim_galore/; last accessed 26 April 2020).

859 Datasets from modENCODE and GEO were aligned to the $d m 6$ Drosophila reference genome (Ensembl

860 release 95) (Cunningham et al. 2019) using STAR (version 2.6.0b) (Dobin et al. 2013) with --

861 chimSegmentMin set to 20. Stringtie (version 1.3.3b) (Pertea et al. 2015) was used to quantify

862 normalized gene expression as Transcripts Per Kilobase Million (TPM) values with the following

863 parameters: -a 5 -e. In addition, the isoform list was provided together with the -G parameter

864 corresponding to the $d m 6$ (Ensembl release 95) GTF file. Genes with TPM values above 2 were

865 considered to be expressed.

866

867

Selection of genes and isoforms for analysis

868

869

Similar to a previous GRO-seq analysis (Core et al. 2008), we used the read density of very

870 large intergenic regions (gene desert regions) to define the reference for absence of transcription. Gene

871 deserts were divided into 50kb windows, and $d$ NET-seq read densities were calculated by dividing the

872 read counts in each window by the window length (in bp). Read counts per window were obtained with

873 bedtools genome coverage (version 2.27.1-1-gb87c465) (Quinlan and Hall 2010), and an arbitrary

874 density threshold was defined as the 90th percentile of the read density distribution (Supplemental Fig

875 S4A). Transcripts whose gene body $d$ NETseq read density exceeded this threshold were considered to

876 be transcriptionally active (Supplemental Fig S4B). For all of the analyses performed on late genes,

877 only transcriptionally active genes were considered. In addition, only one isoform per gene was

878 considered for all analysis, selected as the isoform with the highest RPKM value in the RNA-seq dataset

879 for the corresponding developmental stage. 
bin/hgLiftOver). The most representative isoform for each gene was manually selected through

883

884

885

886

887

888

889

890

891

892

893

894

895

896

897

898

899

900

901

902

903

904

905

906

907

908 visualization of individual profiles.

\section{Splicing intermediate and lariat detection}

Exons containing splicing intermediates or introns containing lariats were identified using a peak finder algorithm (NET_snrPeakFinder) (Churchman and Weissman 2011; Prudêncio et al. 2020) that detects the presence of a peak in the last nucleotide of an exon (splicing intermediate) or an intron (lariat), by comparing the accumulation of 3 ' end reads mapping at that position with the mean read density of the flanking 200 nucleotides. A peak is called when the read density at the peak is superior to the mean of this surrounding region plus 3 standard deviations (Churchman and Weissman 2011). Since gene read density influences peak detection, the exons were divided into quartiles based on the $d$ NET-seq read density of the corresponding gene. Only exons from the highest quartile (i.e. from genes with the highest read density) were considered in Fig $\mathbf{2}$ for $d$ NET-seq Late analysis.

\section{Analysis of read density and peak calling}

RPKM values for the merged $d$ NET-seq datasets were calculated in the following manner:

$$
R P K M(\text { transcript })=\frac{\text { reads } * 10^{4} * 10^{6}}{(\text { total uniquely mapped reads }) *(\text { gene length in } b p)}
$$

Where $10^{4}$ normalizes for gene length and $10^{6}$ normalizes for sequencing depth. Queries of gene 3'UTR overlaps (Figure 3) between genes were performed with bedtools intersect (version 2.27.1-1gb87c465) (Quinlan and Hall 2010).

To detect splicing intermediate and intron lariat peaks, the algorithm from Churchman and Weissman (2011) was adapted as described in Prudêncio et al. (2020). To be able to call larger regions as peaks, a custom algorithm was developed and implemented in Python 3.7. Note that like for all of the custom Python code reported here, there is heavy reliance on bedtools v2.29.0 (Quinlan and Hall 2010) for operations on coordinate intervals. In addition, NumPy v1.17.2 (Harris et al. 2020) and SciPy 
909 1.4.0 (SciPy 1.0 Contributors et al. 2020) were used. Our peak caller detects regions where the local

910 read density is significantly higher than expected by chance given the overall read density of the

911 transcript. Almost all of the numerical parameters used by the peak caller can be adjusted. In Fig $\mathbf{4 A \text { , }}$

912 results obtained with two particular parameterizations are shown. Peak Caller 1 ("large peaks") is

913 adapted to detecting larger peaks and provides results that are more intuitive to a human observer. Peak

914 Caller 2 ("small peaks") provides a finer spatial resolution, and corresponds to the settings used in all

915 of the analyses in this study. The peak caller takes as input a BED file with the 3' ends of reads (single-

916 nucleotide resolution), as well as a GTF file with transcript and exon annotations and a list of transcripts

917 to analyse. It functions by calculating a sliding average of read density within each transcript (window

918 size 5/21 for small/large; only reads mapping to the same strand as the annotated transcript are

919 considered). It then randomly shuffles the positions of the reads within the transcript and recalculates

920 the sliding averages to determine the random expectation. This can be repeated several times (5 in this study) for more robustness. Windows obtained with the true read distribution are called as significant

922 if their read density is higher than the $99^{\text {th }}$ percentile of the simulated windows. Note that in this study,

923 we used a setting whereby this threshold is calculated separately for each exon (and its upstream intron,

924 for exons other than the first), by excluding the intron-exon pair of interest and reads overlapping it

925 during the simulation step. This is necessary so that when calling peaks within a given exon (and its upstream intron), the threshold set would not be affected by the reads within that particular exon and its upstream intron. This way, for instance, the calling of a peak in the beginning of the exon is not affected by the calling of a peak in the middle of the same exon (except through potential merging, see below). After the initial peaks are called, they are filtered to remove peaks where more than $90 \%$ of the reads come from a single nucleotide (probable PCR duplicates), that are shorter than 5 nucleotides, or that overlap with fewer reads than a specified threshold (10/5 for large/small). Finally, peaks that are within a specified distance of each-other (21/5 nucleotides for large/small) are merged together. 


\section{Individual gene profiles}

938

Individual dNET-seq gene profiles were generated by separating reads by strand using SAMtools (version 1.7) (Li et al. 2009). Strand-separated read data was converted to bedGraph format

Coverage values were normalized per nucleotide accounting for the total number of uniquely aligned reads and with the scale set to reads per $10^{8}$ sequences. The outcome was converted to bigwig files through the bedGraphToBigWig tool (Kent et al. 2010) and uploaded to the UCSC genome browser

945 (James Kent et al. 2002).

\section{Metagene analysis}

The read density metagene plots in Fig 3F-I and Fig 5E,F were created with deepTools (version 3.0.2) (Ramírez et al. 2016). Metagenes with normalized gene size (Figure 3) have bins of $10 \mathrm{bp}$ while all other metagene plots in this study have single nucleotide resolution. Normalized gene and intron lengths (Fig 3F-I) were obtained through the scale-regions option. Exon-intron junctions without normalized lengths were obtained using the reference-option set to the 3'SS or the 5'SS. For normalization, we divided the number of reads at each nucleotide (or bin) by the total number of reads in the entire genomic region under analysis. These values were then used to calculate the mean for each nucleotide, and the results were plotted in an arbitrary units (A.U.) ranging from 0 to 1 .

958 S7A-G and S7E-J were prepared using custom Python and $\mathrm{R}$ scripts

959 (https://github.com/rosinaSav/dNETseq_code). The peak density value represents the proportion of introns/exons that overlap with a peak at that position. Only internal fully coding exons that were at least 100 nucleotides long were included. In addition, the intron just upstream of the exon had to be at least 50 nt long. For Fig 7D, S7A-G and S7E-H, further filtering based on read coverage was performed

963 (see below). Note that exons shorter than 150 nucleotides contribute both to the upstream and 964 downstream exonic proportion of the plot. 
Immediate splicing analysis

966 previously annotated pre-MBT genes (Chen et al. 2013) were analysed for early data sets. Reads were considered as spliced if they contained 'N's in the CIGAR string, in a position corresponding to an annotated intron. Reads that overlapped both the (unspliced) intron and the downstream exon were considered as unspliced. In both cases, only reads where the 3' end was located at least 5 nt downstream

972 of the 3' ss were included, to avoid analysing misaligned reads whose 3' end should have mapped to

973 the end of the upstream exon instead. Spliced reads that had the 5' end mapped to the upstream exon

974 and the 3 ' end mapped to the intron were considered indicative of recursive splicing if the first nucleotide of the downstream end (indicative of the ratchet point position) matched the second $\mathrm{G}$ in the AGGT canonical splicing motif. If the last nucleotide of a read matched the last nucleotide of an exon, it was considered a splicing intermediate read and not representative of nascent RNA. A splicing ratio

978 was calculated by dividing the number of nascent RNA spliced reads by the sum of the number of 979 spliced and unspliced nascent RNA reads, only including reads whose 3' ends mapped to the first 100 nt of the downstream exon. Only fully coding internal exons at least $100 \mathrm{nt}$ long were considered (exceptionally, in Fig 6E and $\mathbf{K}$ and Supplemental Fig S6O-Q, the 3' most coding exon was also analysed). Finally, we performed filtering to remove exons where the read coverage was too low to allow for robust estimation of the splicing ratio. The relevant threshold was calculated for each dataset separately. We calculated the total proportion of spliced reads out of all spliced/unspliced reads for the dataset to obtain the expected splicing ratio. We then performed a binomial test to know the probability of sampling only spliced/unspliced reads by chance under the null that the true splicing ratio equalled this expectation. We set the threshold as the lowest number of reads that had to be sampled for the probability to be below 0.01 . Through his procedure, the threshold was set at $>=10$ reads for replicate 1 and 2 of the late data set (no terminal coding exons), at $>=11 / 10$ reads for replicate $1 / 2$ of the late data set including terminal coding exons, and at $>=14 / 9$ reads for replicate $1 / 2$ of the early data set (terminal coding exons always included). 


\section{Gene architecture and nucleotide composition analysis}

994

Gene architecture and nucleotide composition parameters were calculated using custom Python

996

997

998

999

1000

1001

1002

1003

1004

1005

1006

1007

1008

1009

1010

1011

1012

1013

1014

1015

1016

1017

1018

1019

1020

Data access

All raw and processed sequencing data generated in this study have been submitted to the NCBI Gene Expression Omnibus (GEO; https://www.ncbi.nlm.nih.gov/geo/) under accession number GSE152585. The Python code used is available at https://github.com/rosinaSav/dNETseq code and https://github.com/kennyrebelo. The Python code can also be found in the Supplemental Code file.

\section{Competing interests}

The authors declare that they have no competing interests.

\section{Acknowledgements}

We thank Takayuki Nojima and Nicholas Proudfoot (University of Oxford, UK) for critical discussion. This work was supported by funding to M.C.-F. (Fundação para a Ciência e Tecnologia, FCT/ Ministério da Ciência, Tecnologia e Ensino Superior - Fundos do Orçamento de Estado (UIDB/50005/2020), and FCT/FEDER/POR Lisboa 2020, Programa Operacional Regional de Lisboa PORTUGAL 2020, grant LISBOA-01-0145-FEDER-016394) and to R.G.M (FCT grant PTDC/BIA- 
1021 BID/28441/2017). P.P. was a recipient of a FCT fellowship (SFRH/BD/109689/2015). R.S. was a

1022 recipient of an EMBO Long-Term Fellowship (EMBO ALTF 101-2019). This project has received

1023 funding from the European Union's Horizon 2020 research and innovation programme under grant

1024 agreements No 842695 (Marie Skłodowska-Curie Actions) and No 857119 (RiboMed).

1025

1026 Author Contributions

1027

1028 P.P. performed all molecular biology experiments. R.S. performed the majority of splicing kinetics

1029 analyses and developed the peak caller for detecting putative pause sites. K.R. performed additional

1030 bioinformatics analyses. P.P., R.S., R.G.M. and M.C.-F conceived the study and wrote the paper.

1031

1032 
bioRxiv preprint doi: https://doi.org/10.1101/2020.11.05.367888; this version posted July 25, 2021. The copyright holder for this preprint (which was not certified by peer review) is the author/funder, who has granted bioRxiv a license to display the preprint in perpetuity. It is made available under aCC-BY-NC-ND 4.0 International license.

1033

1034

1035

1036

1037

1038

1039

1040

1041

1042 
REFERENCES

1044

1045

1046

1047

1048

1049

1050

1051

1052

1053

1054

1055

1056

1057

1058

1059

1060

1061

1062

1063

1064

1065

1066

1067

1068

1069

1070

1071

1072

1073

1074

1075

1076

1077

1078

Akhtar J, Kreim N, Marini F, Mohana G, Brüne D, Binder H, Roignant J-Y. 2019. Promoterproximal pausing mediated by the exon junction complex regulates splicing. Nat Commun 10: 521.

Alexander RD, Innocente SA, Barrass JD, Beggs JD. 2010. Splicing-dependent RNA polymerase pausing in yeast. Molecular Cell 40: 582-593.

Ali-Murthy Z, Lott SE, Eisen MB, Kornberg TB. 2013. An Essential Role for Zygotic Expression in the Pre-Cellular Drosophila Embryo. PLoS Genetics 9.

Alpert T, Herzel L, Neugebauer KM. 2017. Perfect timing: splicing and transcription rates in living cells. WIRES RNA 8.

Ameur A, Zaghlool A, Halvardson J, Wetterbom A, Gyllensten U, Cavelier L, Feuk L. 2011. Total RNA sequencing reveals nascent transcription and widespread cotranscriptional splicing in the human brain. Nature Structural and Molecular Biology 18: $1435-1440$.

Artieri CG, Fraser HB. 2014. Transcript length mediates developmental timing of gene expression across drosophila. Molecular Biology and Evolution 31: 2879-2889.

Arzate-Mejía RG, Josué Cerecedo-Castillo A, Guerrero G, Furlan-Magaril M, Recillas-Targa F. 2020. In situ dissection of domain boundaries affect genome topology and gene transcription in Drosophila. Nat Commun 11: 894.

Aslanzadeh V, Huang Y, Sanguinetti G, Beggs JD. 2018. Transcription rate strongly affects splicing fidelity and cotranscriptionality in budding yeast. Genome Research 28: 203213.

Bembom O. 2019. seqLogo: Sequence logos for DNA sequence alignments. R package version 1.52.0.

Berget SM. 1995. Exon Recognition in Vertebrate Splicing. The Journal of Biological Chemistry 270: 2411-2414.

Beyer AL, Osheim YN. 1988. Splice site selection, rate of splicing, and alternative splicing on nascent transcripts. Genes \& Development 2: 754-765.

Blythe SA, Wieschaus EF. 2015. Coordinating Cell Cycle Remodeling with Transcriptional Activation at the Drosophila MBT. 1st ed. Elsevier Inc.

Blythe SA, Wieschaus EF. 2016. Establishment and maintenance of heritable chromatin structure during early Drosophila embryogenesis. Elife $\mathbf{5}$.

Boija A, Mahat DB, Zare A, Holmqvist P-H, Philip P, Meyers DJ, Cole PA, Lis JT, Stenberg P, Mannervik M. 2017. CBP Regulates Recruitment and Release of PromoterProximal RNA Polymerase II. Molecular Cell 68: 491-503.e5. 
Brugiolo M, Herzel L, Neugebauer KM. 2013. Counting on co-transcriptional splicing. F1000Prime Reports 5.

Burnette JM, Miyamoto-Sato E, Schaub MA, Conklin J, Lopez AJ. 2005. Subdivision of large introns in Drosophila by recursive splicing at nonexonic elements. Genetics 170: $661-674$.

Bushnell B, Rood J, Singer E. 2017. BBMerge - Accurate paired shotgun read merging via overlap. PLOS ONE 12.

Campos-Ortega JA. 1985. Genetics of early neurogenesis in Drosophila melanogaster. Trends in Neurosciences 8: 245-250.

Carrillo Oesterreich F, Herzel L, Straube K, Hujer K, Howard J, Neugebauer KM. 2016. Splicing of Nascent RNA Coincides with Intron Exit from RNA Polymerase II. Cell 165: 372-381.

Carrillo Oesterreich F, Preibisch S, Neugebauer KM. 2010. Global analysis of nascent RNA reveals transcriptional pausing in terminal exons. Molecular Cell 40: 571-581.

Chathoth KT, Barrass JD, Webb S, Beggs JD. 2014. A Splicing-Dependent Transcriptional Checkpoint Associated with Prespliceosome Formation. Molecular Cell 53: 779-790.

Chen K, Johnston J, Shao W, Meier S, Staber C, Zeitlinger J. 2013. A global change in RNA polymerase II pausing during the Drosophila midblastula transition. eLife 2013.

Churchman LS, Weissman JS. 2011. Nascent transcript sequencing visualizes transcription at nucleotide resolution. Nature 469: 368-373.

Core LJ, Waterfall JJ, Lis JT. 2008. Nascent RNA Sequencing Reveals Widespread Pausing and Divergent Initiation. Science 322: 1845-1849.

Cunningham F, Achuthan P, Akanni W, Allen J, Amode MR, Armean IM, Bennett R, Bhai J, Billis K, Boddu S, et al. 2019. Ensembl 2019. Nucleic Acids Research 47: D745D751.

Dahlberg O, Shilkova O, Tang M, Holmqvist P-H, Mannervik M. 2015. P-TEFb, the Super Elongation Complex and Mediator Regulate a Subset of Non-paused Genes during Early Drosophila Embryo Development ed. G.P. Copenhaver. PLOS Genetics 11: e1004971.

David CJ, Boyne AR, Millhouse SR, Manley JL. 2011. The RNA polymerase II C-terminal domain promotes splicing activation through recruitment of a U2AF65-Prp19 complex. Genes and Development 25: 972-982.

de Almeida SF, Carmo-Fonseca M. 2008. The CTD role in cotranscriptional RNA processing and surveillance. FEBS Letters 582: 1971-1976.

De Conti L, Baralle M, Buratti E. 2013. Exon and intron definition in pre-mRNA splicing. Wiley interdisciplinary reviews RNA 4: 49-60. 
De Renzis S, Elemento O, Tavazoie S, Wieschaus EF. 2007. Unmasking activation of the zygotic genome using chromosomal deletions in the Drosophila embryo. PLoS Biology 5: 1036-1051.

Dingwall C, Lomonossoff GP, Laskey RA. 1981. High sequence specificity of micrococcal nuclease. Nucleic Acids Research 9: 2659-2674.

Dinno A. 2017. dunn.test: Dunn's Test of Multiple Comparisons Using Rank Sums. R package version 1.3.5.

Dobin A, Davis CA, Schlesinger F, Drenkow J, Zaleski C, Jha S, Batut P, Chaisson M, Gingeras TR. 2013. STAR: Ultrafast universal RNA-seq aligner. Bioinformatics 29: $15-21$.

Drexler HL, Choquet K, Churchman LS. 2020. Splicing Kinetics and Coordination Revealed by Direct Nascent RNA Sequencing through Nanopores. Molecular Cell 77: 985998.e8.

Duff MO, Olson S, Wei X, Garrett SC, Osman A, Bolisetty M, Plocik A, Celniker SE, Graveley BR. 2015. Genome-wide identification of zero nucleotide recursive splicing in Drosophila. Nature 521: 376-379.

Emili A, Shales M, McCracken S, Xie W, Tucker PW, Kobayashi R, Blencowe BJ, Ingles CJ. 2002. Splicing and transcription-associated proteins PSF and $\mathrm{p} 54 \mathrm{nrb} / \mathrm{NonO}$ bind to the RNA polymerase II CTD. Rna 8: 1102-1111.

Erickson JW, Cline TW. 1993. A bZIP protein, sisterless-a, collaborates with bHLH transcription factors early in Drosophila development to determine sex. Genes and Development 7: 1688-1702.

Fong N, Kim H, Zhou Y, Ji X, Qiu J, Saldi T, Diener K, Jones K, Fu XD, Bentley DL. 2014. Pre-mRNA splicing is facilitated by an optimal RNA polymerase II elongation rate. Genes and Development 28: 2663-2676.

Fox-Walsh KL, Dou Y, Lam BJ, Hung S -p., Baldi PF, Hertel KJ. 2005. The architecture of pre-mRNAs affects mechanisms of splice-site pairing. Proceedings of the National Academy of Sciences 102: 16176-16181.

Fukaya T, Lim B, Levine M. 2017. Rapid Rates of Pol II Elongation in the Drosophila Embryo. Current Biology 27: 1387-1391.

Gaffney DJ, McVicker G, Pai AA, Fondufe-Mittendorf YN, Lewellen N, Michelini K, Widom J, Gilad Y, Pritchard JK. 2012. Controls of Nucleosome Positioning in the Human Genome. PLoS Genetics 8.

Gelfman S, Cohen N, Yearim A, Ast G. 2013. DNA-methylation effect on cotranscriptional splicing is dependent on GC architecture of the exon-intron structure. Genome Research 23: 789-799.

Görnemann J, Barrandon C, Hujer K, Rutz B, Rigaut G, Kotovic KM, Faux C, Neugebauer KM, Séraphin B. 2011. Cotranscriptional spliceosome assembly and splicing are independent of the Prp40p WW domain. Rna 17: 2119-2129. 
Graveley BR, Brooks AN, Carlson JW, Duff MO, Landolin JM, Yang L, Artieri CG, Baren MJ Van, Boley N, Booth BW, et al. 2011. The Developmental Transcriptome of Drosophila melanogaster. Nature 471: 473-479.

Guilgur LG, Prudêncio P, Sobral D, Liszekova D, Rosa A, Martinho RG. 2014. Requirement for highly efficient pre-mRNA splicing during Drosophila early embryonic development. eLife 3 .

Harris CR, Millman KJ, van der Walt SJ, Gommers R, Virtanen P, Cournapeau D, Wieser E, Taylor J, Berg S, Smith NJ, et al. 2020. Array programming with NumPy. Nature 585: $357-362$.

Hatton AR, Subramaniam V, Lopez AJ. 1998. Generation of alternative Ultrabithorax isoforms and stepwise removal of a large intron by resplicing at exon-exon junctions. Molecular Cell 2: 787-796.

Herzel L, Straube K, Neugebauer KM. 2018. Long-read sequencing of nascent RNA reveals coupling among RNA processing events. 1008-1019.

Heyn P, Kircher M, Dahl A, Kelso J, Tomancak P, Kalinka AT, Neugebauer KM. 2014. The earliest transcribed zygotic genes are short, newly evolved, and different across species. Cell Reports 6: 285-292.

Hörz W, Altenburger W. 1981. Sequence specific cleavage of DNA by micrococcal nuclease. Nucleic Acids Research 9: 2643-2658.

Hoskins RA, Landolin JM, Brown JB, Sandler JE, Takahashi H, Lassmann T, Booth BW, Zhang D, Wan KH, Yang L, et al. 2011. Genome-wide analysis of promoter architecture in Drosophila melanogaster. Genome Research 21: 182-192.

Hsin JP, Manley JL. 2012. The RNA polymerase II CTD coordinates transcription and RNA processing. Genes and Development 26: 2119-2137.

James Kent W, Sugnet CW, Furey TS, Roskin KM, Pringle TH, Zahler AM, Haussler D. 2002. The human genome browser at UCSC. Genome Research 12: 996-1006.

Ji J-Y, Squirrell JM, Schubiger G. 2004. Both Cyclin B levels and DNA-replication checkpoint control the early embryonic mitoses in Drosophila Jun-Yuan. Development 131: 401-411.

Jonkers I, Kwak H, Lis JT. 2014. Genome-wide dynamics of Pol II elongation and its interplay with promoter proximal pausing, chromatin, and exons. eLife 2014.

Joseph B, Kondo S, Lai EC. 2018. Short cryptic exons mediate recursive splicing in Drosophila. Nature Structural and Molecular Biology 25: 365-371.

Kent WJ, Zweig AS, Barber G, Hinrichs AS, Karolchik D. 2010. BigWig and BigBed: Enabling browsing of large distributed datasets. Bioinformatics 26: 2204-2207.

Keren H, Lev-Maor G, Ast G. 2010. Alternative splicing and evolution: Diversification, exon definition and function. Nature Reviews Genetics 11: 345-355. 
bioRxiv preprint doi: https://doi.org/10.1101/202011.05.367888; this version posted July 25,2021 . The copyright holder for this preprin (which was not certified by peer review) is the author/funder, who has granted bioRxiv a license to display the preprint in perpetuity. It is made available under aCC-BY-NC-ND 4.0 International license.

1191

1192

1193

1194

1195

1196

1197

1198

1199

1200

1201

1202

1203

1204

1205

1206

1207

1208

1209

1210

1211

1212

1213

1214

1215

1216

1217

1218

1219

1220

1221

1222

1223

1224

1225

1226

1227

1228

1229

Khodor YL, Menet JS, Tolan M, Rosbash M. 2012. Cotranscriptional splicing efficiency differs dramatically between Drosophila and mouse. RNA 18: 2174-2186.

Khodor YL, Rodriguez J, Abruzzi KC, Tang CHA, Marr MT, Rosbash M. 2011. Nascent-seq indicates widespread cotranscriptional pre-mRNA splicing in Drosophila. Genes and Development 25: 2502-2512.

Kimura H, Tao Y, Roeder RG, Cook PR. 1999. Quantitation of RNA Polymerase II and Its Transcription Factors in an HeLa Cell: Little Soluble Holoenzyme but Significant Amounts of Polymerases Attached to the Nuclear Substructure. Molecular and Cellular Biology 19: 5383-5392.

Kwak H, Fuda NJ, Core LJ, Lis JT. 2013. Precise maps of RNA polymerase reveal how promoters direct initiation and pausing. Science 339: 950-953.

Kwasnieski JC, Orr-Weaver TL, Bartel DP. 2019. Early genome activation in Drosophila is extensive with an initial tendency for aborted transcripts and retained introns. Genome Research 29: 1188-1197.

Larson MH, Mooney RA, Peters JM, Windgassen T, Nayak D, Gross CA, Block SM, Greenleaf WJ, Landick R, Weissman JS. 2014. A pause sequence enriched at translation start sites drives transcription dynamics in vivo. Science 344: 1042-1047.

Laver JD, Marsolais AJ, Smibert CA, Lipshitz HD. 2015. Regulation and Function of Maternal Gene Products During the Maternal-to-Zygotic Transition in Drosophila. 1st ed. Elsevier Inc.

Lee JEA, Mitchell NC, Zaytseva O, Chahal A, Mendis P, Cartier-Michaud A, Parsons LM, Poortinga G, Levens DL, Hannan RD, et al. 2015. Defective Hfp-dependent transcriptional repression of $\mathrm{dMYC}$ is fundamental to tissue overgrowth in Drosophila XPB models. Nat Commun 6: 7404.

Li H, Handsaker B, Wysoker A, Fennell T, Ruan J, Homer N, Marth G, Abecasis G, Durbin R. 2009. The Sequence Alignment/Map format and SAMtools. Bioinformatics 25: 2078-2079.

Lott SE, Villalta JE, Schroth GP, Luo S, Tonkin LA, Eisen MB. 2011. Noncanonical compensation of zygotic $\mathrm{X}$ transcription in early Drosophila melanogaster development revealed through single-embryo RNA-Seq. PLoS Biology 9.

Lund E, Dahlberg JE. 1992. Cyclic 2', 3 “-Phosphates and Nontemplated Nucleotides at the 3" End of Spliceosomal U6 Small Nuclear RNAs. Science 255: 327-330.

Martin M. 2011. Cutadapt removes adapter sequences from high-throughput sequencing reads. EMBnet.journal 17: 10-12.

Martin RM, Rino J, Carvalho C, Kirchhausen T, Carmo-Fonseca M. 2013. Live-Cell Visualization of Pre-mRNA Splicing with Single-Molecule Sensitivity. Cell Reports 4: 1144-1155.

Martinho RG, Guilgur LG, Prudêncio P. 2015. How gene expression in fast-proliferating cells keeps pace. BioEssays 37: 514-524. 
Maslon MM, Braunschweig U, Aitken S, Mann AR, Kilanowski F, Hunter CJ, Blencowe BJ, Kornblihtt AR, Adams IR, Cáceres JF. 2019. A slow transcription rate causes embryonic lethality and perturbs kinetic coupling of neuronal genes. The EMBO Journal 38.

Mayer A, Di Iulio J, Maleri S, Eser U, Vierstra J, Reynolds A, Sandstrom R, Stamatoyannopoulos JA, Churchman LS. 2015. Native elongating transcript sequencing reveals human transcriptional activity at nucleotide resolution. Cell 161: $541-554$.

Morris DP, Greenleaf AL. 2000. The splicing factor, Prp40, binds the phosphorylated carboxyl-terminal domain of RNA Polymerase II. Journal of Biological Chemistry 275: 39935-39943.

Nojima T, Gomes T, Carmo-Fonseca M, Proudfoot NJ. 2016. Mammalian NET-seq analysis defines nascent RNA profiles and associated RNA processing genome-wide. Nature Protocols 11: 413-428.

Nojima T, Gomes T, Grosso ARF, Kimura H, Dye MJ, Dhir S, Carmo-Fonseca M, Proudfoot NJ. 2015. Mammalian NET-seq reveals genome-wide nascent transcription coupled to RNA processing. Cell 161: 526-540.

Nojima T, Rebelo K, Gomes T, Grosso AR, Proudfoot NJ, Carmo-Fonseca M. 2018. RNA Polymerase II Phosphorylated on CTD Serine 5 Interacts with the Spliceosome during Co-transcriptional Splicing. Molecular Cell 72: 369-379.e4.

Pai AA, Henriques T, McCue K, Burkholder A, Adelman K, Burge CB. 2017. The kinetics of pre-mRNA splicing in the Drosophila genome and the influence of gene architecture. eLife 6.

Pai AA, Paggi JM, Yan P, Adelman K, Burge CB. 2018. Numerous recursive sites contribute to accuracy of splicing in long introns in flies. PLoS Genetics 14.

Pertea M, Pertea GM, Antonescu CM, Chang TC, Mendell JT, Salzberg SL. 2015. StringTie enables improved reconstruction of a transcriptome from RNA-seq reads. Nature Biotechnology 33: 290-295.

Pritchard DK, Schubiger G. 1996. Activation of transcription in Drosophila embryos is a gradual process mediated by the nucleocytoplasmic ratio. Genes and Development 10: $1131-1142$.

Prudêncio P, Rebelo K, Grosso AR, Martinho RG, Carmo-fonseca M. 2020. Analysis of Mammalian Native Elongating Transcript sequencing (mNET-seq)high-throughput data. Methods 178: 89-95.

Quinlan AR, Hall IM. 2010. BEDTools: A flexible suite of utilities for comparing genomic features. Bioinformatics 26: 841-842.

Ramírez F, Ryan DP, Grüning B, Bhardwaj V, Kilpert F, Richter AS, Heyne S, Dündar F, Manke T. 2016. deepTools2: a next generation web server for deep-sequencing data analysis. Nucleic acids research 44: W160-W165. 
bioRxiv preprint doi: https://doi org/10.1101/2020.11.05.367888; this version posted July 25,2021 . The copyright holder for this preprin (which was not certified by peer review) is the author/funder, who has granted bioRxiv a license to display the preprint in perpetuity. It is made available under aCC-BY-NC-ND 4.0 International license.

Reimer KA, Mimoso CA, Adelman K, Neugebauer KM. 2021. Co-transcriptional splicing regulates 3' end cleavage during mammalian erythropoiesis. Molecular Cell 81: 9981012.e7.

Robberson BL, Cote GJ, Berget SM. 1990. Exon Definition May Facilitate Splice Site Selection in RNAs with Multiple Exons. Molecular and Cellular Biology 10: 84-94.

Rothe M, Pehl M, Taubert H, Jäckle H. 1992. Loss of gene function through rapid mitotic cycles in the Drosophila embryp. Nature 359: 156-159.

Sandler JE, Irizarry J, Stepanik V, Dunipace L, Amrhein H, Stathopoulos A. 2018. A Developmental Program Truncates Long Transcripts to Temporally Regulate Cell Signaling. Developmental Cell 47: 773-784.e6.

Saunders A, Core LJ, Sutcliffe C, Lis JT, Ashe HL. 2013. Extensive polymerase pausing during Drosophila axis patterning enables high-level and pliable transcription. Genes and Development 27: 1146-1158.

Schlackow M, Nojima T, Gomes T, Dhir A, Carmo-Fonseca M, Proudfoot NJ. 2017. Distinctive Patterns of Transcription and RNA Processing for Human lincRNAs. Molecular Cell 65: 25-38.

Schulz KN, Bondra ER, Moshe A, Villalta JE, Lieb JD, Kaplan T, McKay DJ, Harrison MM. 2015. Zelda is differentially required for chromatin accessibility, transcription factor binding, and gene expression in the early Drosophila embryo. Genome Res 25: 17151726.

SciPy 1.0 Contributors, Virtanen P, Gommers R, Oliphant TE, Haberland M, Reddy T, Cournapeau D, Burovski E, Peterson P, Weckesser W, et al. 2020. SciPy 1.0: fundamental algorithms for scientific computing in Python. Nat Methods 17: 261272.

Sheridan RM, Fong N, D’Alessandro A, Bentley DL. 2019. Widespread Backtracking by RNA Pol II Is a Major Effector of Gene Activation, 5' Pause Release, Termination, and Transcription Elongation Rate. Molecular Cell 73: 107-118.e4.

Shermoen AW, O'Farrell PH. 1991. Progression of the Cell Cycle through Mitosis Leads to Abortion of Nascent Transcripts. Cell 67: 303-310.

Sterner DA, Carlo T, Berget SM. 1996. Architectural limits on split genes. Proc Natl Acad Sci USA 93: 15081-15085.

Veloso A, Kirkconnell KS, Magnuson B, Biewen B, Paulsen MT, Wilson TE, Ljungman M. 2014. Rate of elongation by RNA polymerase II is associated with specific gene features and epigenetic modifications. Genome Research 24: 896-905.

Vizcaya-Molina E, Klein CC, Serras F, Mishra RK, Guigó R, Corominas M. 2018. Damageresponsive elements in Drosophila regeneration. Genome Res 28: 1852-1866.

Windhager L, Bonfert T, Burger K, Ruzsics Z, Krebs S, Kaufmann S, Malterer G, L'Hernault A, Schilhabel M, Schreiber S, et al. 2012. Ultrashort and progressive 4sU-tagging 

Research 22: 2031-2042.

Yan D, Neumüller RA, Buckner M, Ayers K, Li H, Hu Y, Yang-Zhou D, Pan L, Wang X, Kelley C, et al. 2014. A Regulatory Network of Drosophila Germline Stem Cell SelfRenewal. Developmental Cell 28: 459-473.

Yeo G, Burge CB. 2004. Maximum entropy modeling of short sequence motifs with applications to RNA splicing signals. Journal of Computational Biology 11: 377-394.

1314 Yuan K, Seller CA, Shermoen AW, Farrell PHO. 2016. Timing the Drosophila Mid-Blastula Transition: A Cell Cycle-Centered View. Trends in Genetics 32: 496-507.

Zhu J, Liu M, Liu X, Dong Z. 2018. RNA polymerase II activity revealed by GRO-seq and pNET-seq in Arabidopsis. Nature Plants 4: 1112-1123. architecture variation of genes in eukaryotic genomes. BMC Genomics $\mathbf{1 0 .}$ 
bioRxiv preprint doi: https://doi.org/10.1101/2020.11.05.367888; this version posted July 25, 2021. The copyright holder for this preprint (which was not certified by peer review) is the author/funder, who has granted bioRxiv a license to display the preprint in perpetuity. It is made available under aCC-BY-NC-ND 4.0 International license.

\section{SUPPLEMENTAL FIGURES}


bioRxiv preprint doi: https://doi.org/10.1101/2020.11.05.367888; this version posted July 25, 2021. The copyright holder for this preprint (which was not certified by peer review) is the author/funder, who has granted bioRxiv a license to display the preprint in perpetuity. It is made available under aCC-BY-NC-ND 4.0 International license.

A

\begin{tabular}{|c|c|c|}
\hline Dataset & Raw reads & Uniquely Mapped after Merge \\
\hline Early_2-3h_S5P_rep1 & $62,891,575$ & $12,959,070$ \\
\hline Early_2-3h_S5P_rep2 & $56,917,697$ & $3,610,607$ \\
\hline Late_4-6h_S5P_rep1 & $65,865,258$ & $21,564,673$ \\
\hline Late_4-6h_S5P_rep2 & $75,909,131$ & $27,375,998$ \\
\hline Late_4-6h_S5P_rep3 & $71,923,876$ & $11,987,034$ \\
\hline Late_4-6h_S2P_rep1 & $77,838,463$ & $38,595,780$ \\
\hline Late_4-6h_S2P_rep2 & $64,862,996$ & $23,137,529$ \\
\hline Late_4-6h_S2P_rep3 & $76,719,886$ & $17,912,504$ \\
\hline
\end{tabular}

B

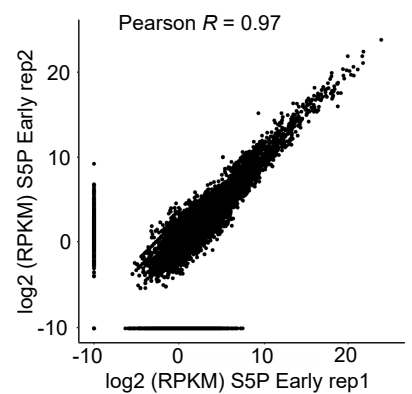

C

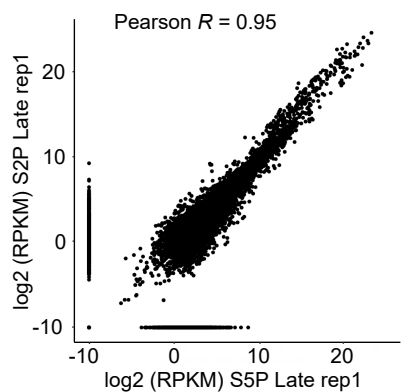

D

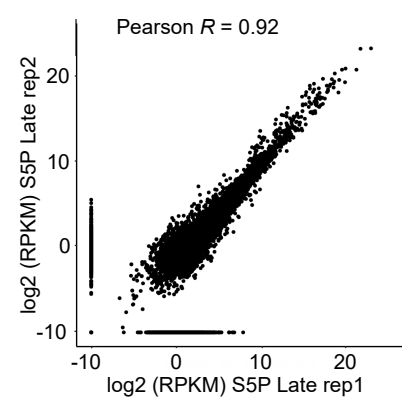

E

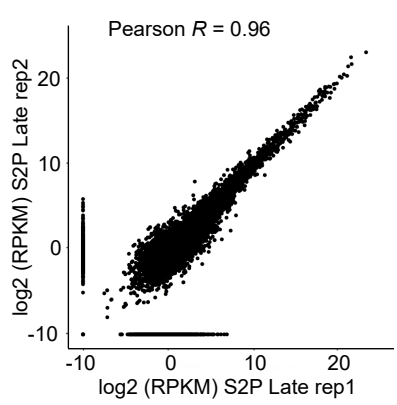

F

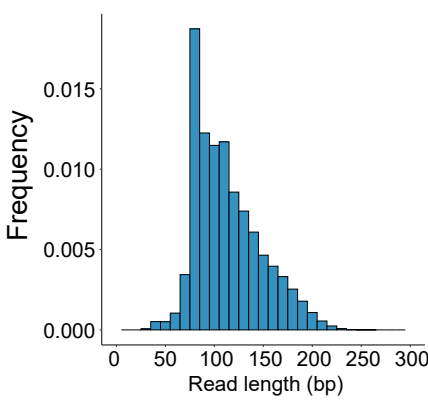

G

\begin{tabular}{|c|c|c|}
\hline Dataset & Number of nascent reads & Nascent reads / Uniquely mapped reads (\%) \\
\hline Early_2-3h_S5P_rep1 & $7,984,411$ & 61.6 \\
\hline Early_2-3h_S5P_rep2 & $2,430,876$ & 67.3 \\
\hline Late_4-6h_S5P_rep1 & $13,413,045$ & 62.2 \\
\hline Late_4-6h_S5P_rep2 & $13,777,880$ & 50.3 \\
\hline Late_4-6h_S5P_rep3 & $8,505,056$ & 71.0 \\
\hline Late_4-6h_S2P_rep1 & $23,153,659$ & 60.0 \\
\hline Late_4-6h_S2P_rep2 & $12,926,707$ & 55.9 \\
\hline Late_4-6h_S2P_rep3 & $10,356,743$ & 57.8 \\
\hline
\end{tabular}

$\mathrm{H}$

\begin{tabular}{|c|c|c|c|c|c|c|}
\hline Dataset & min & mean & max & 25th percentile & 50th percentile & 75th percentile \\
\hline Early_2-3h_S5P_rep1 & 35 & 99.6 & 293 & 74 & 81 & 119 \\
\hline Early_2-3h_S5P_rep2 & 35 & 94.3 & 293 & 72 & 81 & 110 \\
\hline Late_4-6h_S5P_rep1 & 35 & 114.2 & 293 & 86 & 108 & 135 \\
\hline Late_4-6h_S5P_rep2 & 35 & 102.0 & 294 & 80 & 87 & 119 \\
\hline Late_4-6h_S5P_rep3 & 35 & 88.4 & 294 & 76 & 81 & 89 \\
\hline Late_4-6h_S2P_rep1 & 35 & 107.0 & 294 & 81 & 96 & 128 \\
\hline Late_4-6h_S2P_rep2 & 35 & 111.1 & 294 & 81 & 102 & 132 \\
\hline Late_4-6h_S2P_rep3 & 35 & 109.0 & 293 & 83 & 105 & 130 \\
\hline
\end{tabular}


Supplemental Fig. S1. (A) For each $d$ NET-seq library prepared, the number of total reads and of uniquely aligned reads is indicated. (B) Density of uniquely aligned reads per gene (RPKM in $\log 2$ scale) for two $d$ NET-seq/S5P biological replicates from early embryos (Pearson's correlation, R=0.97). (C) Density of uniquely aligned reads per gene (RPKM in log2 scale) from late embryos analysed by $d$ NET-seq/S5P and $d$ NET-seq/S2P (Pearson's correlation, R = 0.95). (D, E) Density of uniquely aligned reads per gene (RPKM in $\log 2$ scale) for two $d$ NET-seq/S5P and $d$ NET-seq/S2P biological replicates from late embryos (Pearson's correlation, $\mathrm{R}=0.92 ; \mathrm{R}=0.96$ ). (F) Histogram of the read lengths in $d$ NET-seq/S5P data from late embryos. (G) For each $d$ NET-seq library generated, the number of uniquely aligned reads corresponding to nascent transcripts is indicated. (H) Length of sequenced nascent RNA (in nucleotides). 


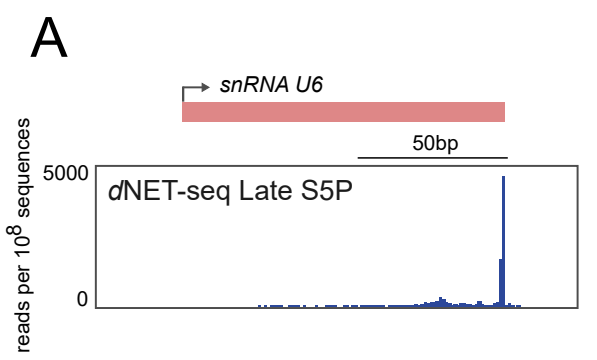

B

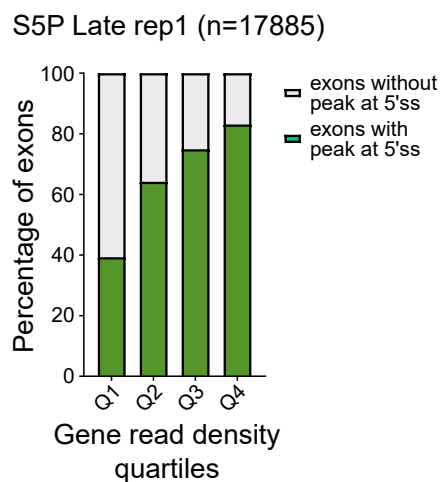

Supplemental Fig. S2. (A) $d$ NET-seq/S5P profile over the U6 snRNA gene in the late dataset (replicate 1). (B) Frequency of peaks corresponding to splicing intermediates (green) detected by $d$ NET-seq/S5Pon exons of genes expressed in late embryos. Genes were grouped into quartiles (Q) based on their $d$ NET-seqread density. 
bioRxiv preprint doi: https://doi.org/10.1101/2020.11.05.367888; this version posted July 25, 2021. The copyright holder for this preprint (which was not certified by peer review) is the author/funder, who has granted bioRxiv a license to display the preprint in perpetuity. It is made available under aCC-BY-NC-ND 4.0 International license.

A

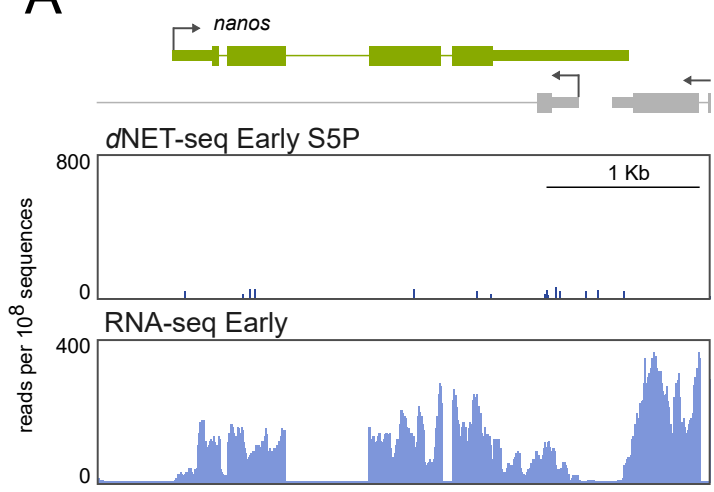

C

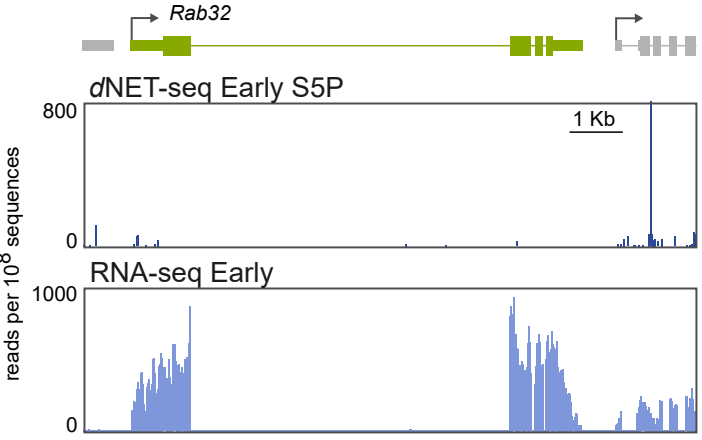

E

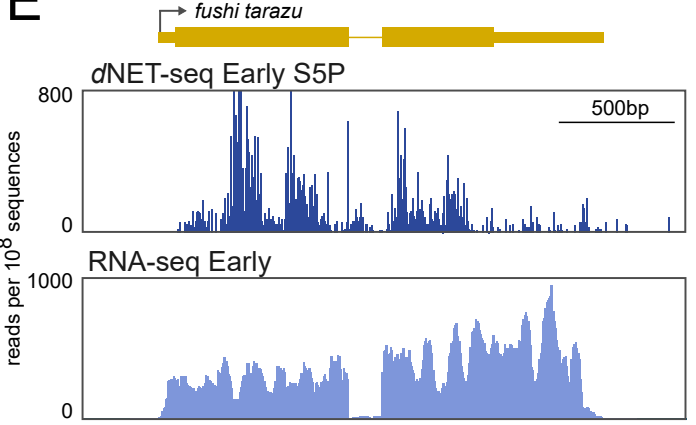

B

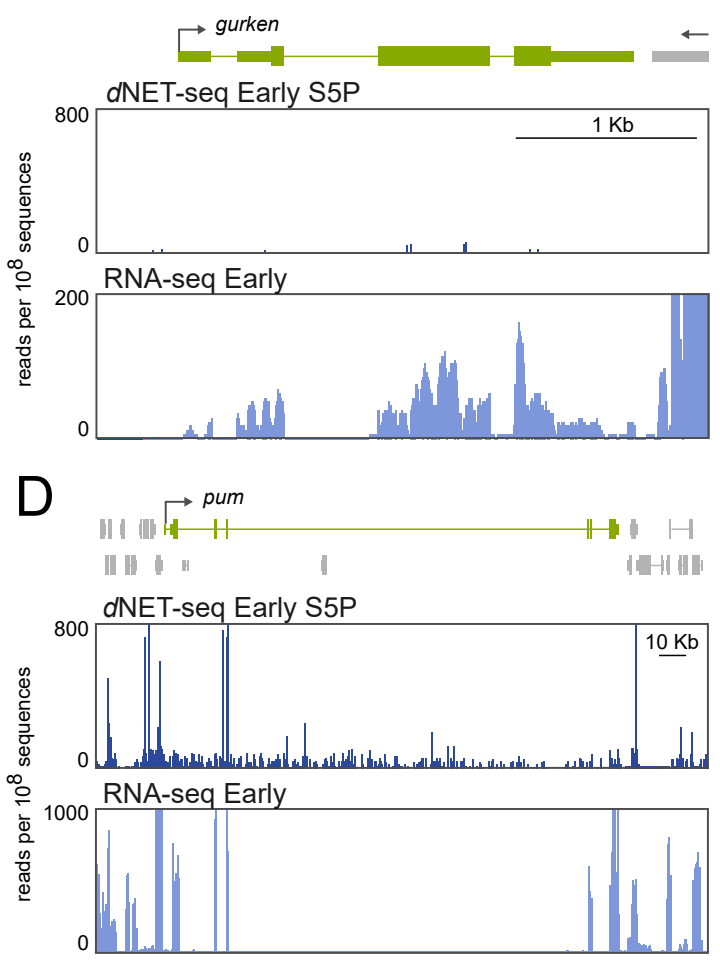

$\mathrm{F}$

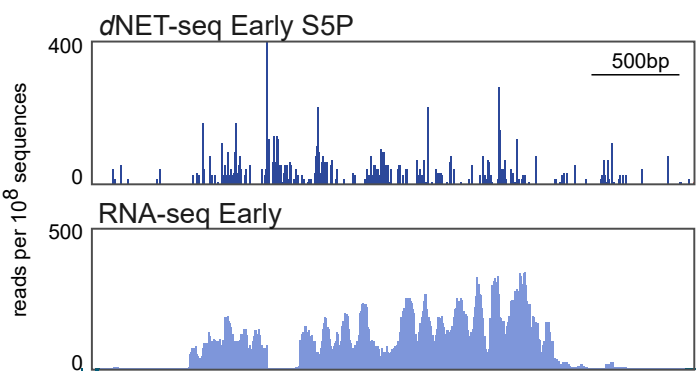

G

S5P early replicate 2

$\mathrm{H}$

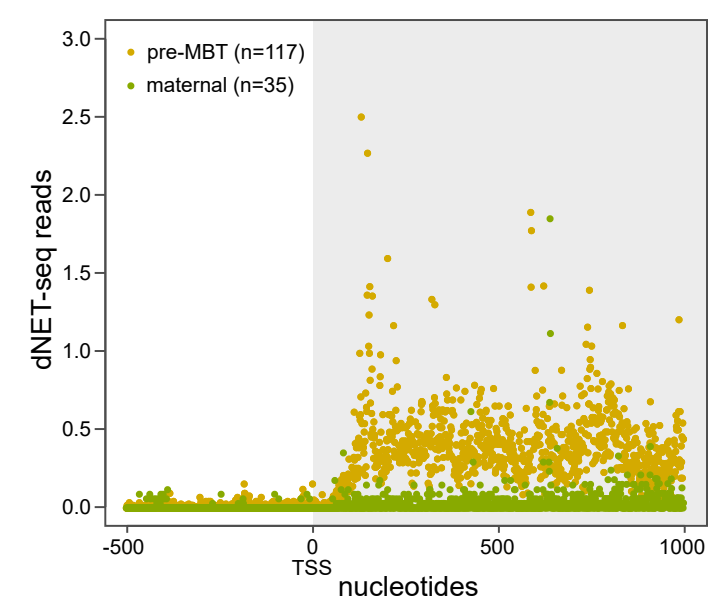

$\longrightarrow$ His $3.3 A$

$\longrightarrow$ His3.3A

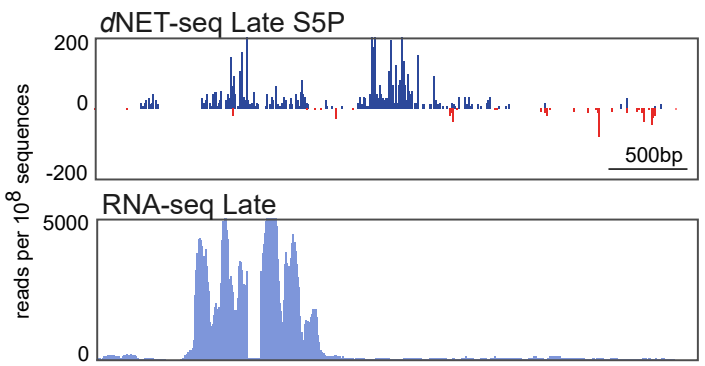

$\Gamma^{t s r}$

पrit-

IntS1

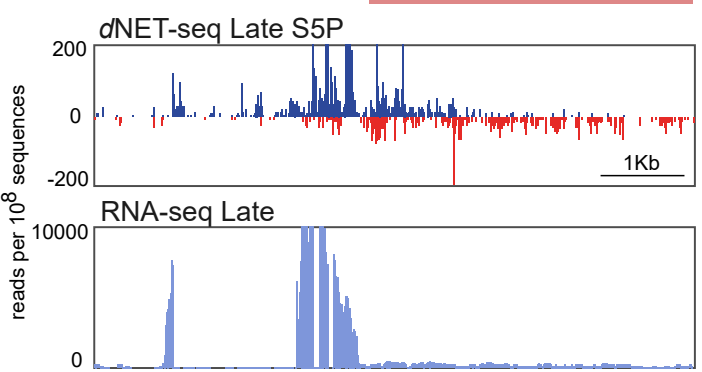


bioRxiv preprint doi: https://doi.org/10.1101/2020 11.05.367888; this version posted July 25, 2021. The copyright holder for this preprint

Supplemental Fig. S3. (A-D) $d$ NET-seq/S5P and RNA-seq profiles over maternal genes in early embryos: nanos (A), gurken (B), Rab32 (C) and pumilio (pum) (D). (E-F) $d$ NET-seq/S5P and RNAseq profiles over pre-MBT genes in early embryos: fushi tarazu (E) and odd skipped (odd) (F). (G) Meta-analysis of $d$ NET-seq/S5P mean read density around the transcription start site (TSS) in maternal and pre-MBT genes (replicate 2). (H-I) $d$ NET-seq/S5P and RNA-seq profiles over MBT genes in late embryos: His3.3A (H) and twinstar (tsr) (I). Reads that aligned to the positive strand are in blue, and reads that aligned to the negative strand are in red. The direction of transcription is indicated by an arrow. 
bioRxiv preprint doi: https://doi.org/10.1101/2020.11.05.367888; this version posted July 25, 2021. The copyright holder for this preprint (which was not certified by peer review) is the author/funder, who has granted bioRxiv a license to display the preprint in perpetuity. It is made available under aCC-BY-NC-ND 4.0 International license.

A

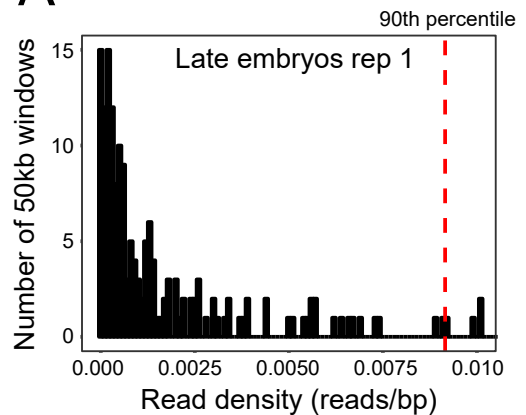

C
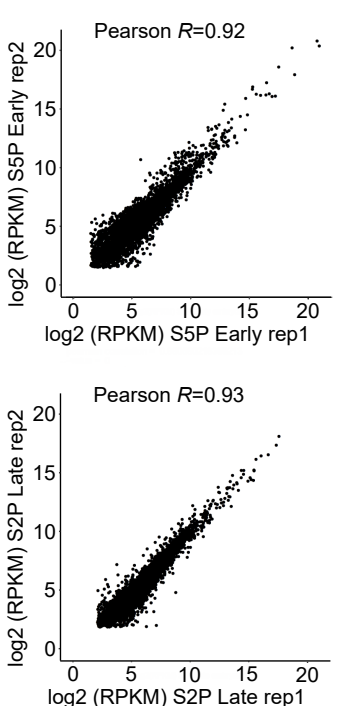

Transcriptionally Active Genes S5P Early rep1 S5P Early rep2 $\mathrm{n}=6892 \quad \mathrm{n}=6805$

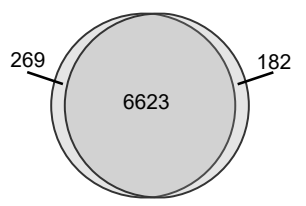

Transcriptionally Active Genes S2P Late rep1 S2P Late rep2 $\mathrm{n}=7237 \quad \mathrm{n}=7045$

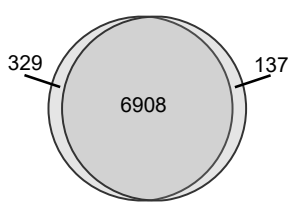

$\mathrm{D}$

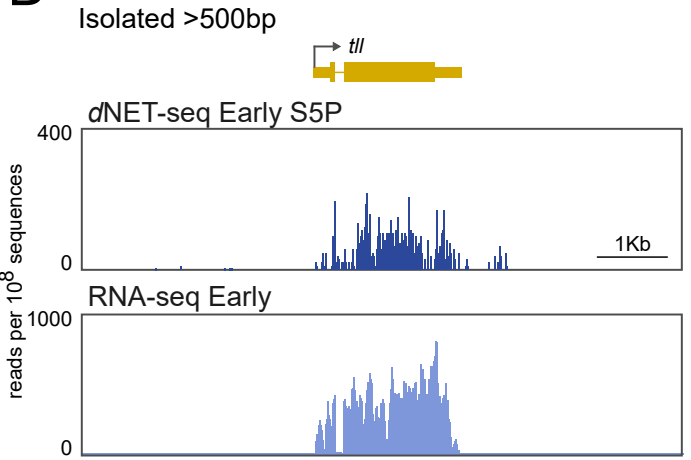

F Proximal $<500 \mathrm{bp}$

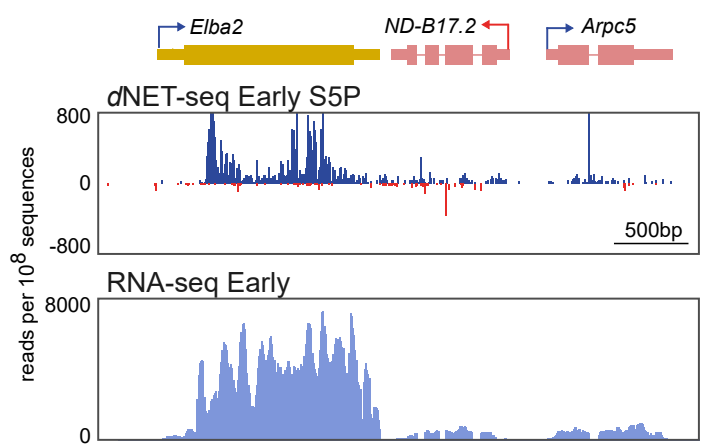

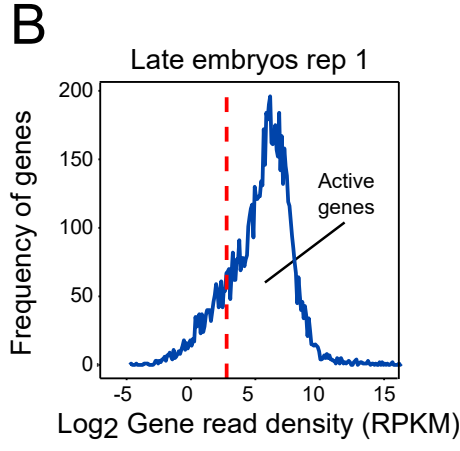

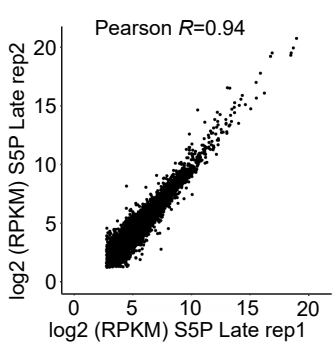

Transcriptionally Active Genes S5P Late rep1 S5P Late rep2 $n=7233 \quad n=7467$
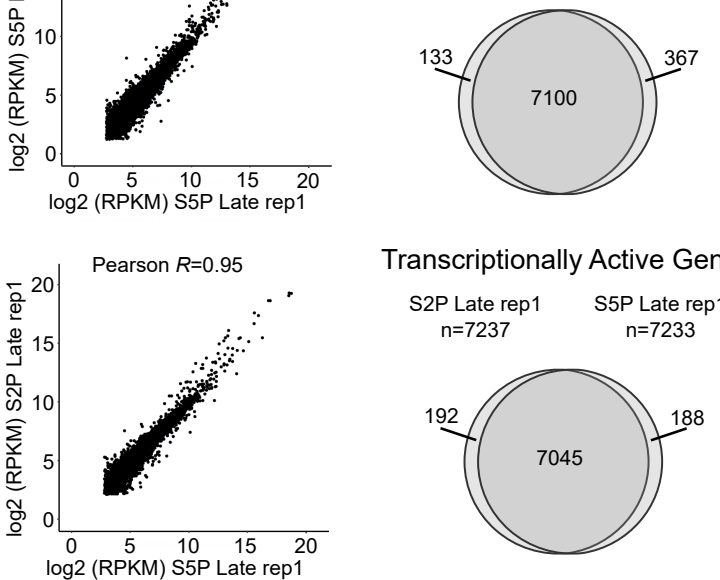

Transcriptionally Active Genes S2P Late rep1 S5P Late rep1 $n=7237 \quad n=7233$

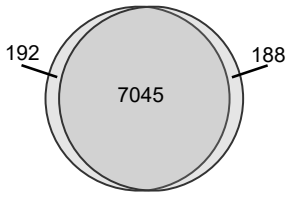

E

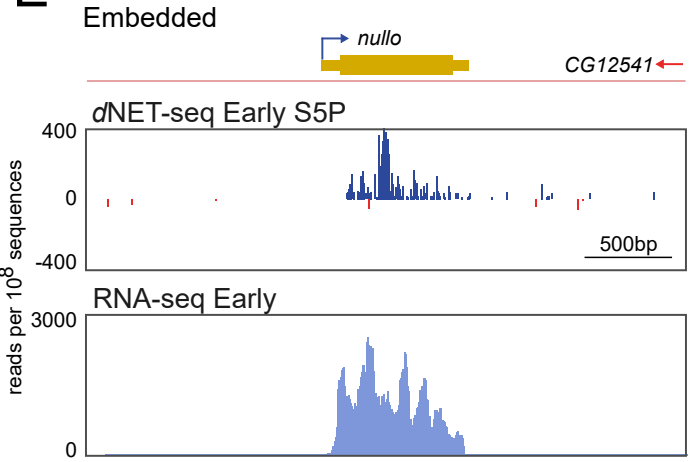

G Overlapped 3'UTR

$\longrightarrow$ spo

I(3)psg2 $\longleftarrow$

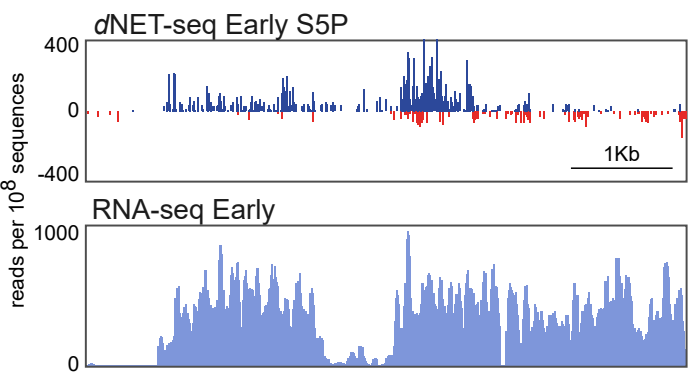


Supplemental Fig. S4. (A-C) Identification of transcriptionally active genes based on $d$ NET-seq signal along the gene body in late embryos. (A) Read density distribution in intergenic regions (gene deserts). The red dashed line represents the 90th percentile of read density in all intergenic regions analysed. (B) Read density distribution per gene (RPKM) represented in Log2 scale. The 90th percentile of read density over gene deserts is set as threshold (red dashed line). (C) Comparison of read density per active gene (RPKM in $\log 2$ scale) between the indicated datasets. Total number of active genes identified in each dataset is indicated $(n)$. Venn diagrams show common active genes between datasets. (D-G) $d$ NET-seq/S5P and RNA-seq profiles over the indicated pre-MBT genes. Reads that aligned to the positive strand are in blue, and reads that aligned to the negative strand are in red. The direction of transcription is indicated by an arrow. 
bioRxiv preprint doi: https://doi.org/10.1101/2020 11.05.367888; this version posted July 25, 2021. The copyright holder for this preprint (which was not certified by peer review) is the author/funder, who has granted bioRxiv a license to display the preprint in perpetuity. It is made available under aCC-BY-NC-ND 4.0 International license.

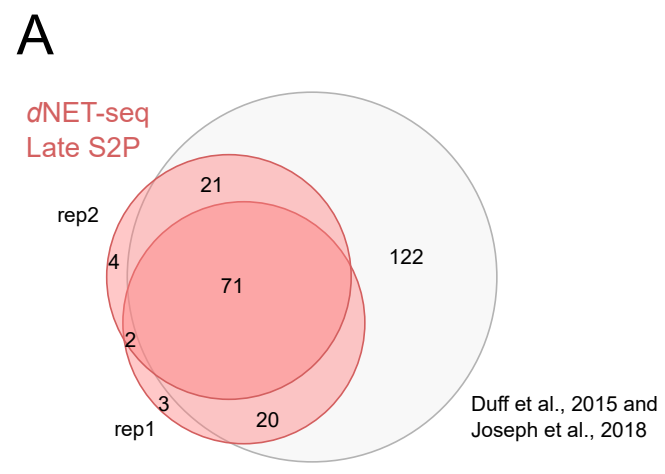

Supplemental Fig . S5. (A) Venn diagram comparing RPs identified in two $d$ NET-seq/S2P biological replicates and in previously reported studies (Joseph et al. 2018; Duff et al. 2015). 
bioRxiv preprint doi: https://doi.org/10.1101/2020.11.05.367888; this version posted July 25, 2021. The copyright holder for this preprint (which was not certified by peer review) is the author/funder, who has granted bioRxiv a license to display the preprint in perpetuity. It is A made aBilable under aCC-BY-NC-ND 4.0 Inte Cational license.
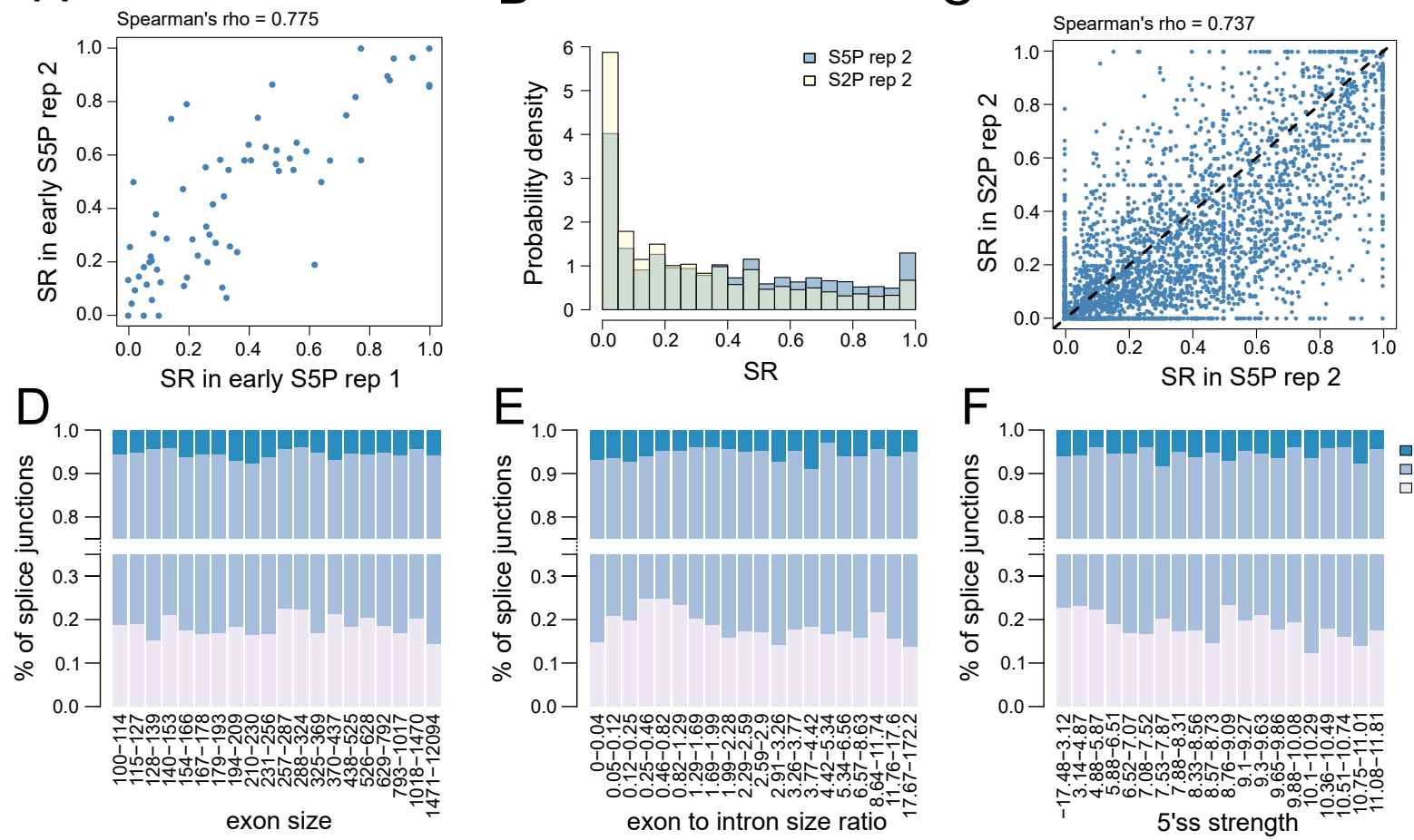

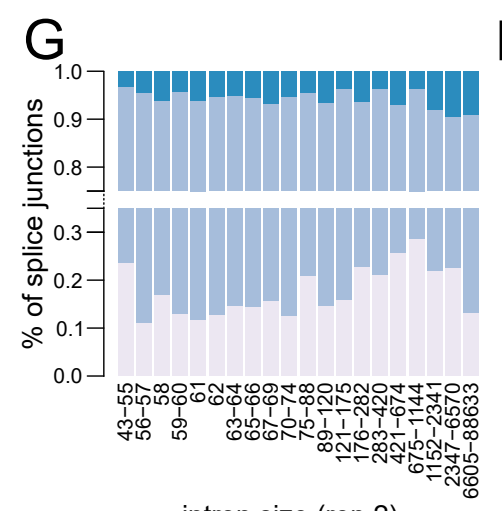

intron size (rep 2)

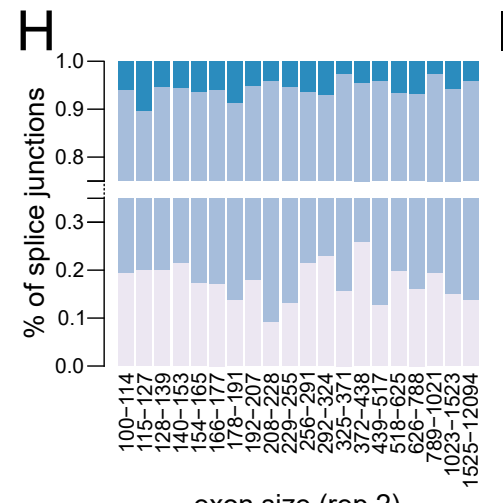

exon size (rep 2)

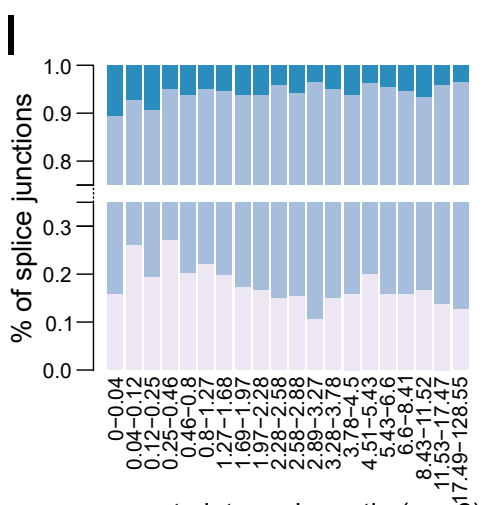

exon to intron size ratio (rep $\overline{2}$ )

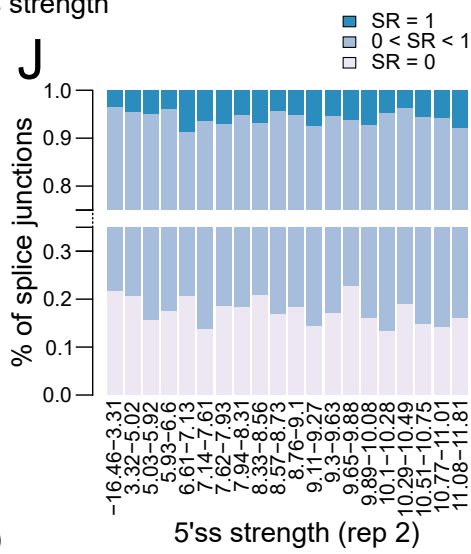

$\mathrm{K}$

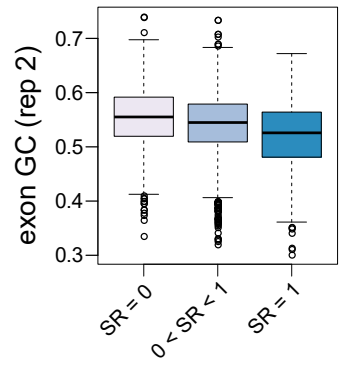

$\mathrm{O}$

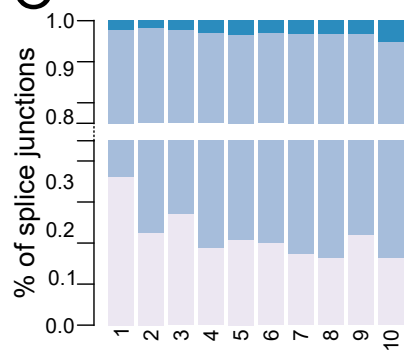

intron number (rep 1)
M

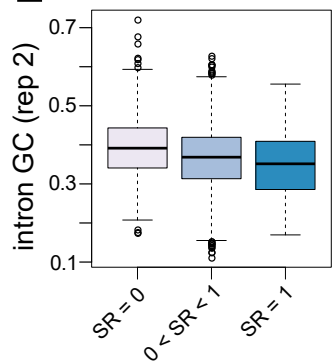

$P$

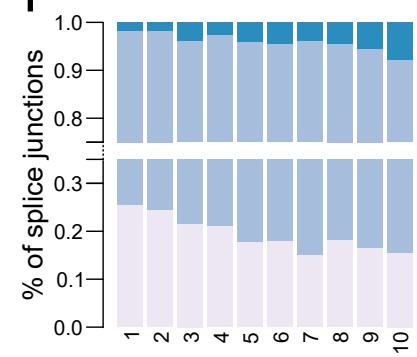

intron number (rep 2)

$\mathrm{R}$

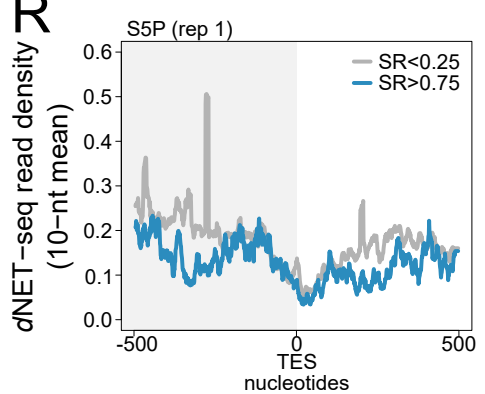

$\mathrm{N}$

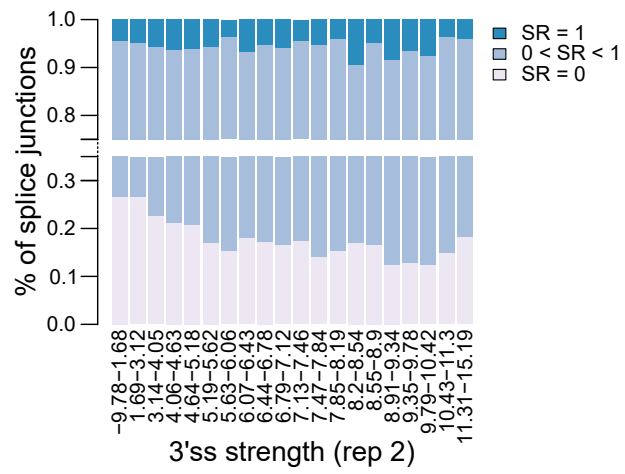

$Q$

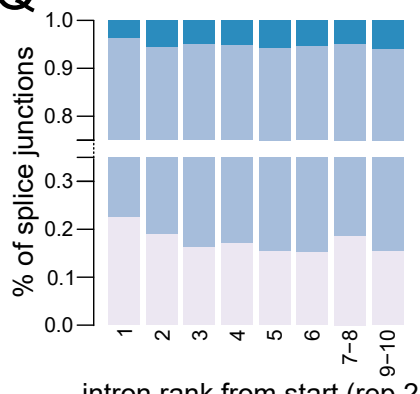

intron rank from start (rep 2)

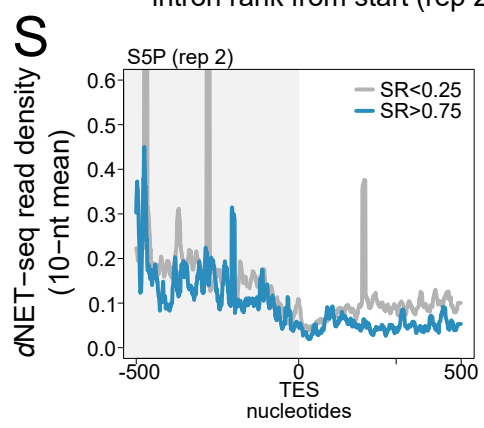

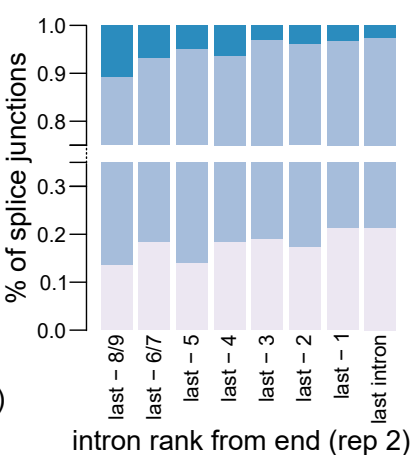

intron rank from end (rep 2) 
Supplemental Fig. S6. In all panels, unless otherwise specified, only introns from transcriptionally active genes where the downstream exon is a fully coding internal exon at least $100 \mathrm{nt}$ long were included. In addition, enough spliced/unspliced reads had to end within the first 100 exonic nucleotides that obtaining a splicing ratio (SR) of 0 or 1 by chance alone was highly unlikely (see the Methods section for details). For genes expressed in late embryos, this threshold was 10 reads for both replicates. For pre-MBT genes, it was 14 for replicate 1 and 9 for replicate 2. For S2P, a threshold of 10 was used for better comparability with S5P data. (A) SR values estimated in two biological replicates of $d$ NETseq/S5P datasets for pre-MBT genes in early embryos (Spearman correlation, $\rho=\sim 0.775, P \sim 1.348 *$ $\left.10^{-14}\right)$. (B) Distribution of SR values for $d$ NET-seq/S5P $(\mathrm{N}=4510)$ and $d$ NET-seq/S2P $(\mathrm{N}=4373)$. (C) SR values estimated in replicate 2 of $d$ NET-seq/S5P and $d$ NET-seq/S2P from late embryos (Spearman correlation, $\rho=\sim 0.737, P<2.2 * 10^{-16} ; \mathrm{N}=3295$ ). (D-Q) SR values for $d$ NET-seq/S5P data (replicate 1 and 2) plotted out against several gene architecture parameters. For sample sizes and statistical tests, see Supplementary Table 1. In (D-J, N-Q), the bin ranges have been set so that intron numbers would be as equal possible between bins. (R-S) $d$ NET-seq/S5P read density around the transcription end site (TES) (averaged over a 10-nucleotide sliding window) for genes with different mean splicing ratios in replicate $1(\mathbf{R})$ and $2(\mathbf{S})$. There is no significant correlation between SR and the count of reads 101 300 nucleotides past the TES (partial Spearman correlation controlling for the count of reads in the region 500 to 301 nucleotides before the TES as a proxy for gene body rad density; replicate 1: $\rho=$ $\sim 0.013, P=\sim 0.519 ; \mathrm{N}=2450$; replicate $2: \rho=\sim-0.034, \mathrm{P}=\sim 0.126 ; \mathrm{N}=2052)$. 
bioRxiv preprint doi: https://doi.org/10.1101/2020.11.05.367888; this version posted July 25, 2021. The copyright holder for this preprint (which was not certified by peer review) is the author/funder, who has granted bioRxiv a license to display the preprint in perpetuity. It is made available under aCC-BY-NC-ND 4.0 International license.
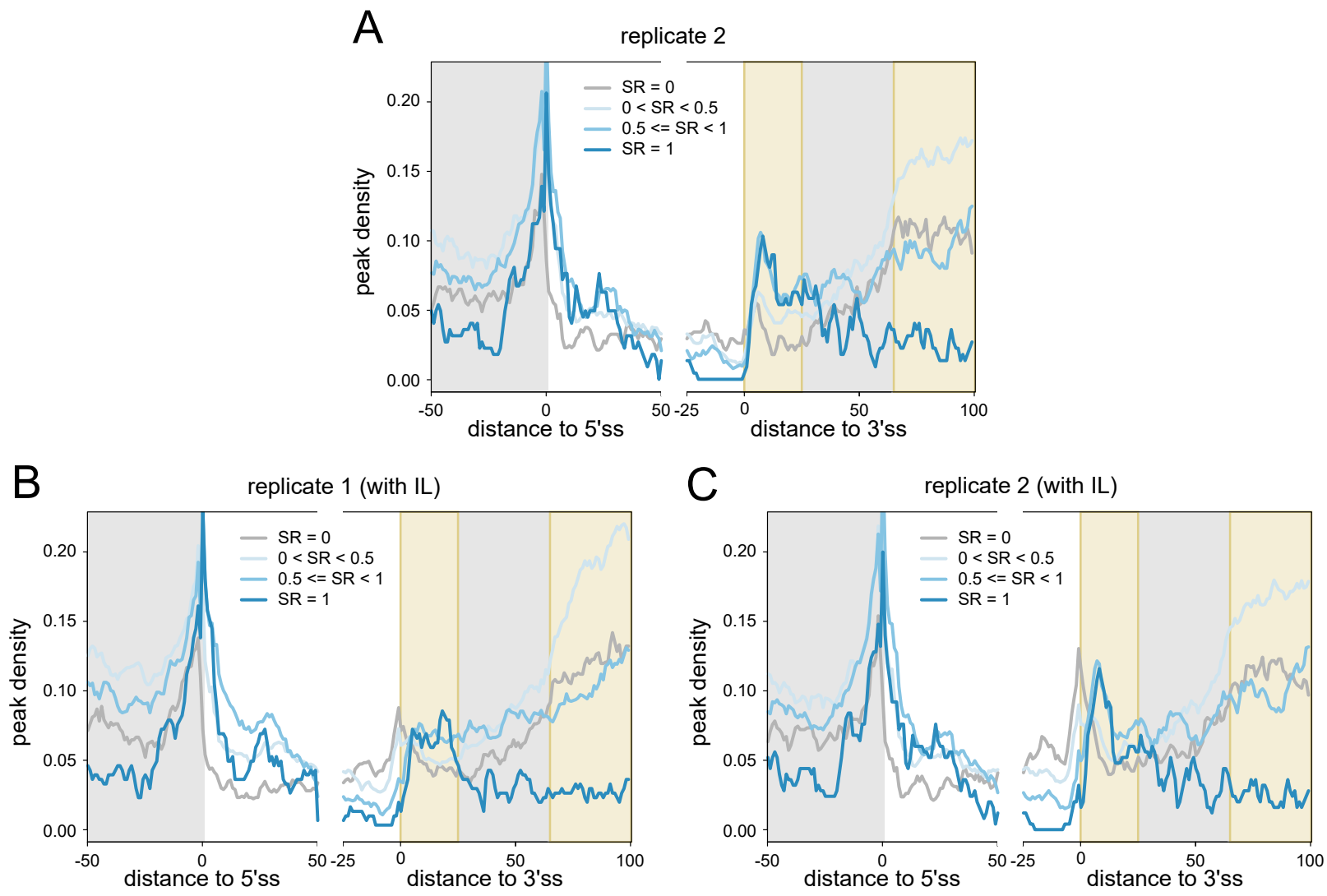

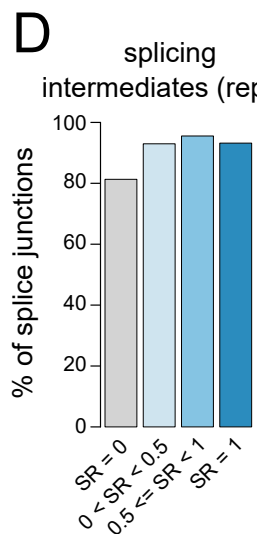

G

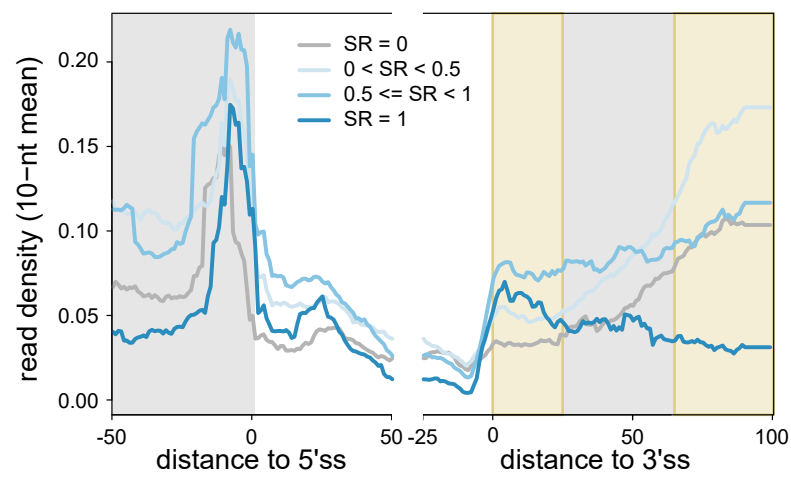

I $\quad$ NET-seq peak density metaprofile S2P rep $1(n=13184)$

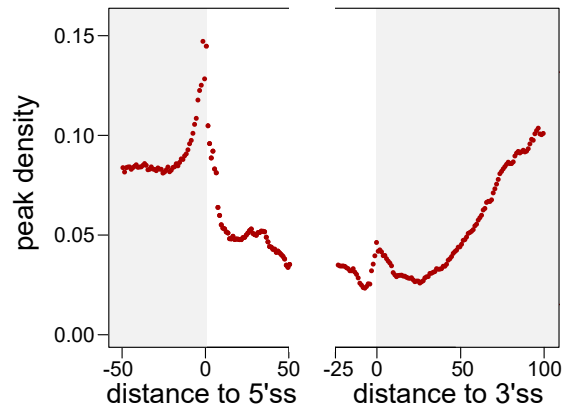

$\mathrm{F}$

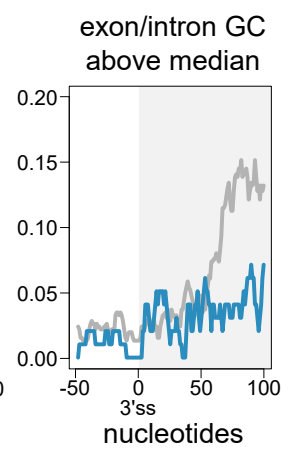

$\mathrm{H}$

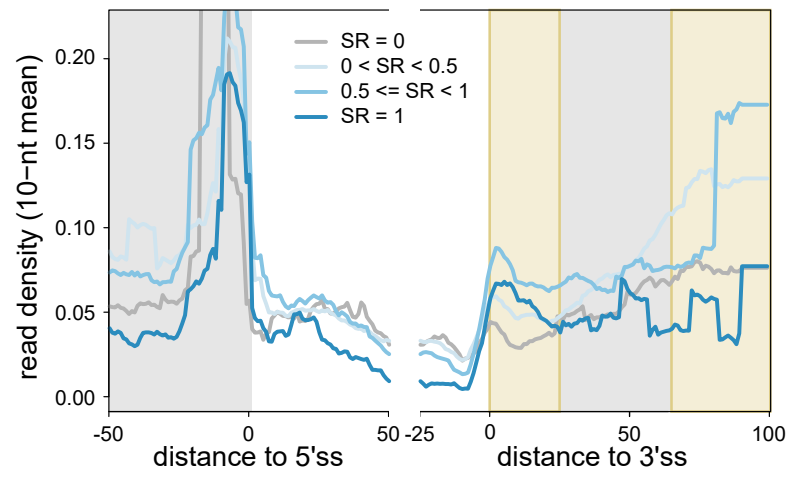

J $\quad$ NNET-seq peak density metaprofile S2P rep $2(n=11742)$

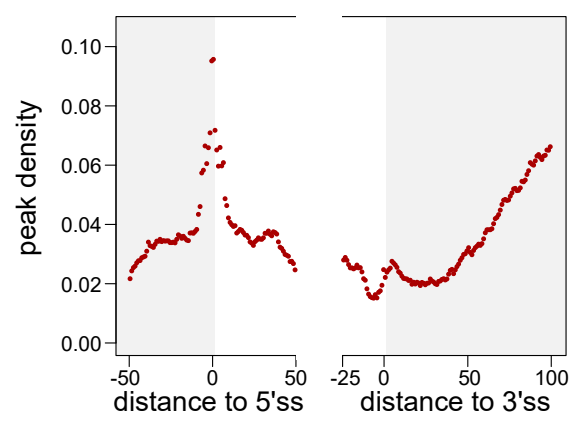


Supplemental Fig. S7. (A-C) Metagene analyses of peak density estimated from $d$ NET-seq/S5P datasets from late embryos for different SR ranges. (A) shows replicate 2, with introns with putative intron lariat reads removed $(\mathrm{N}=3623)$. $(\mathbf{B})$ and $(\mathbf{C})$ show replicates 1 and 2, respectively, with introns with putative intron lariat reads included ( $\mathrm{N}=5614$ for replicate 1 and $\mathrm{N}=4503$ for replicate 2). Peak density has been calculated as the proportion of introns that overlap with a peak at any given position. The last 50 nucleotides of exons, the first 50 nucleotides of introns, the last 25 nucleotides of introns, and the first 100 nucleotides of exons are shown. Only internal and fully coding exons from transcriptionally active genes that are at least 100 nucleotides long are shown. In addition, at least 10 spliced/unspliced reads had to end within the first 100 nucleotides of the exon. (D) The proportion of introns with at least one read whose 3' end maps to the final position of the upstream exon (putative splicing intermediates) or to the final position of the intron (putative intron lariats) in $d$ NET-seq/S5P late replicate 2. (E-F) $d$ NET-seq/S5P peak density profiles for late embryos, replicate $1(\mathbf{E})$ and replicate 2 (F). The final 50 nucleotides of the intron and the first 100 nucleotides of the downstream exon are shown. For each data point, the GC content over the first 100 exonic nucleotides was divided by the GC content over the last 50 intronic nucleotides, generating the exonic to intronic GC ratio. Introns were split into two classes along the median of this metric. Only introns with an SR of 0 or 1 were plotted. (G-H) Similar to 7D and S7A, respectively, but showing read densities (averaged over a 10nucleotide sliding window) rather than peak densities. (I-J) Metagene analysis of peak density estimated from $d$ NET-seq/S2P datasets from late embryos, in replicate 1(I) and replicate $2(\mathbf{J})$. The last 50 nucleotides of exons, the first 50 nucleotides of introns, the last 25 nucleotides of introns, and the first 100 nucleotides of exons are shown. Only internal and fully coding exons from transcriptionally active genes that are at least 100 nucleotides long are shown $(\mathrm{N}=13229$ for replicate $1, \mathrm{~N}=11831$ for replicate 2). Exons shorter than 150 nucleotides contribute to both the exon end and start. Only introns that were at least $50 \mathrm{nt}$ long were considered. 


\section{SUPPLEMENTARY METHODS $d$ NET-seq sequence biases}

Previous work has shown that MNase cleaves DNA more frequently at AT than at GC base pairs (Dingwall et al., 1981). More specifically, Hörz and Altenburger (1981) found a preference for sites where a few alternating AT-TA base pairs were surrounded by a more GC-rich region, rather than for sites within longer AT-rich stretches. More recent genomewide work in humans has come to similar conclusions (Gaffney et al., 2012). dNET-seq relies on MNase cleavage of both DNA and RNA to solubilize chromatin. Any nucleotide biases in cleavage may be problematic, as they could mean that the solubilization is more efficient in regions where the nucleotide composition is more in line with such cleavage biases. This could lead to an artefactually increased read density in such regions.

To verify whether there was any evidence for RNA cleavage biases, we determined the nucleotide composition around the locations of the 5' ends of NET-seq reads (Figure 4D in main text). A clear preference for cleavage just $5^{\prime}$ of an adenine was observed, with additional weaker biases at surrounding sites. Our results are therefore broadly in line with those obtained previously for DNA.

To what extent could these biases affect our results? We generated a simulated version of a $d$ NET-seq dataset (S5P late, replicate 1) where the read positions were randomized in such a way as to preserve the nucleotide biases at the $5^{\prime}$ ends of reads. Only reads that overlapped transcriptionally active genes were considered. More specifically, for each read, we defined the "starting hexamer" as the hexamer centred on the 5' end of the read (the three nucleotides just 5' of the read and the first three nucleotides of the read). We then randomly picked another occurrence of the same hexamers from within transcriptionally active genes and defined a "simulated read" at that position, preserving the length of the initial read. We then called peaks on the simulated reads similarly to true reads.

The mean proportion of nucleotides within simulated peaks was similar for both exons and introns ( 0.017). However, this belies a difference in medians, with a higher peak density in exons than in introns (exon median: $\sim 0.008$; intron median: $0 ; P<2.2 * 10^{-16}$; two-tailed Mann-Whitney $U$-test; Supplementary Methods Figure 1). To know if the 5' end nucleotide bias effect could explain the bias towards exons observed in real data, we calculated a normalized peak density for each exon and intron, as $\frac{\text { true peak density-simulated peak density }}{\text { simulated peak density }}$. Exons and introns with a simulated peak density of 0 were discarded. Normalized peak densities were still higher for exons than for introns $(P<$ $2.2 * 10^{-16}$; two-tailed Mann-Whitney $U$-test). Hence, 5' end nucleotide biases may exaggerate but do not explain the bias towards exons in real data. 

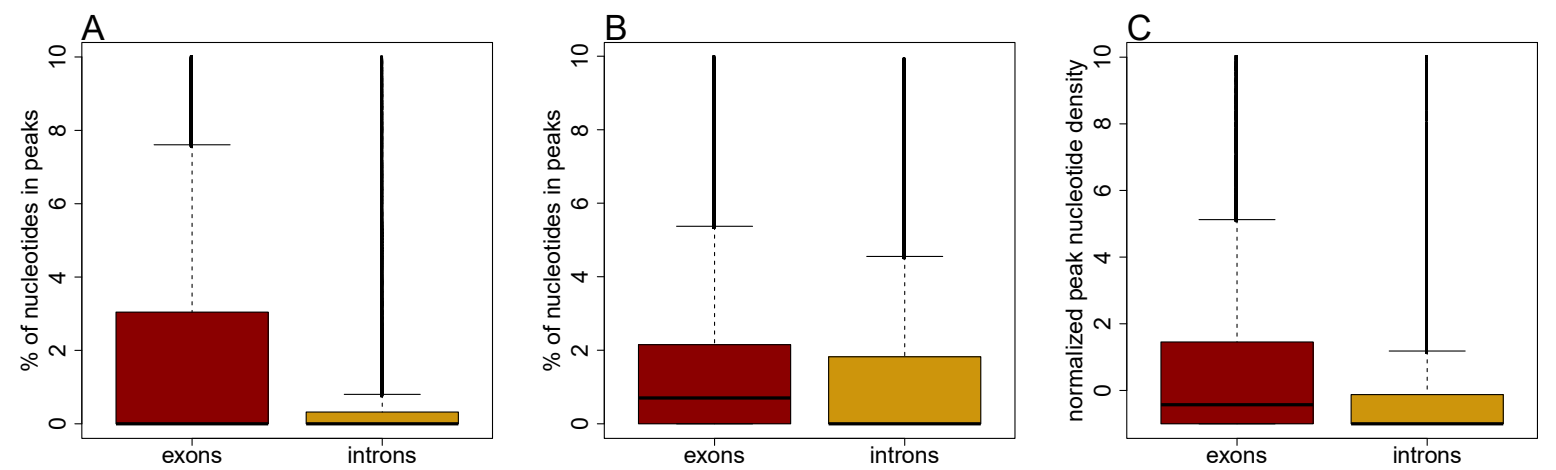

Supplementary Methods Figure 1: distribution of peak densities for exons and introns. A: true peaks. B: simulated peaks. C: normalized peaks, obtained as $\frac{\text { true peak density-simulated peak density }}{\text { simulated peak density }}$. For all three panels, the y axis has been limited to values below 10 for visualization reasons.

It may seem surprising that simulated read density would be higher in exons, given that Drosophila exons tend to have a higher GC content than introns (Zhu et al., 2009). Indeed, one would, at first sight, expect the bias to exaggerate intronic peak density, rather than exonic peak density. However, peaks are called based on the locations of the 3' ends of reads, whereas the bias affects the locations of the 5' ends of reads. Therefore, many reads whose 3' ends are in GC-rich exonic sequence are expected to have their 5' ends in AT-rich intronic sequence.

Further examination of the meta-profiles reveals that the simulated peaks do not display the increased peak density in the beginning of the exons (first highlighted region in Supplementary Methods Figure 2B), as observed for true data (Supplementary Methods Figure 2A). It thus appears that this peak cannot be explained by read 5' end biases. However, the second highlighted region shows increased peak densities both in true and simulated data (Supplementary Methods Figure 2A-B). To verify if the increase observed in this region with true data could be explained by read 5' end nucleotide composition biases, we divided the true data into two classes based on whether or not any simulated peaks mapped to within the second highlighted region (Supplementary Methods Figure 2C-D). There was no visible difference in true mean peak densities between the two groups within the second highlighted region. This suggests that any nucleotide biases acting at the 5' ends of reads are unlikely to explain this increase in peak densities. 

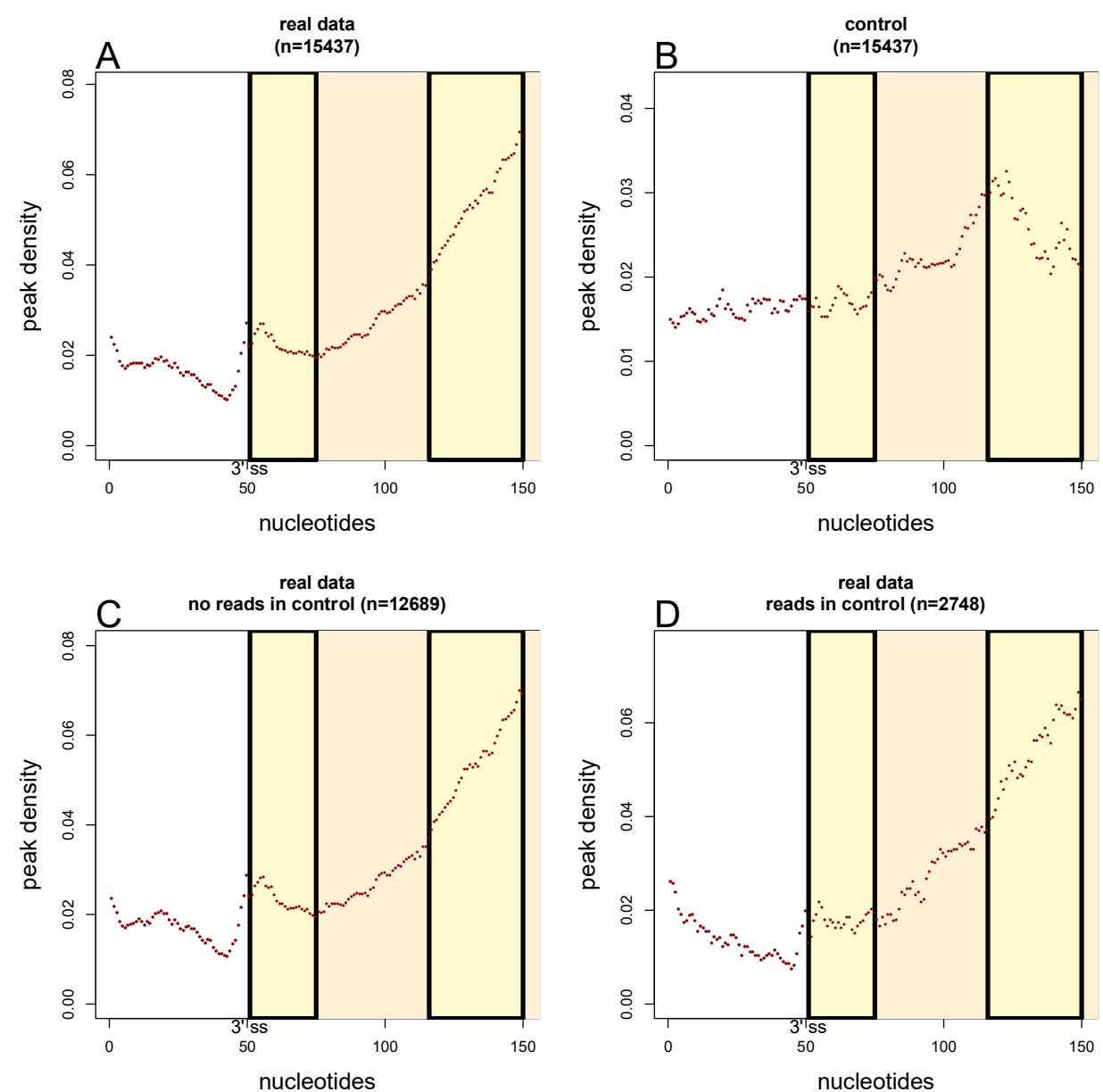

Supplementary Methods Figure 2: average peak density for the last 50 nucleotides of introns and the first 100 nucleotides of exons (concatenated). The orange boxes show the positions of exons. Only exons at least $100 \mathrm{nt}$ long have been included.

Dingwall, C, Lomonossoff, GP, and Laskey, RA (1981). High sequence specificity of micrococcal nuclease. Nucleic Acids Res 9, 2659-2674.

Gaffney, DJ, McVicker, G, Pai, AA, Fondufe-Mittendorf, YN, Lewellen, N, Michelini, K, Widom, J, Gilad, Y, and Pritchard, JK (2012). Controls of Nucleosome Positioning in the Human Genome. PLoS Genet 8, 1-13.

Hörz, W, and Altenburger, W (1981). Sequence specific cleavage of DNA by micrococcal nuclease. Nucleic Acids Res 9, 2643-2658.

Zhu, L, Zhang, Y, Zhang, W, Yang, S, Chen, J, and Tian, D (2009). Patterns of exon-intron architecture variation of genes in eukaryotic genomes. 12, 1-12. 